\title{
A-posteriori reduced basis error-estimates for a semi-discrete in space quasilinear parabolic PDE
}

\author{
Fabian Hoppe $^{1}$ (D) Ira Neitzel $^{1}$
}

Received: 17 December 2020 / Accepted: 28 June 2021

(c) The Author(s) 2021

\begin{abstract}
We prove a-posteriori error-estimates for reduced-order modeling of quasilinear parabolic PDEs with non-monotone nonlinearity. We consider the solution of a semidiscrete in space equation as reference, and therefore incorporate reduced basis-, empirical interpolation-, and time-discretization-errors in our consideration. Numerical experiments illustrate our results.
\end{abstract}

Keywords Quasilinear parabolic partial differential equation · Reduced basis · Proper Orthogonal Decomposition · A-posteriori error

Mathematics Subject Classification 35K59 - 65M15

\section{Introduction}

In the present paper we are concerned with a-posteriori error estimation for model order reduction applied to a semi-discrete in space quasilinear parabolic partial differential equation (PDE) with non-monotone nonlinearity. The PDE appears for instance as state equation in the optimal control problems from [9, 35], and is used in the modeling of heat conduction, when the thermal conductivity of the material under consideration is temperature-dependent, cf. e.g. [48, 49, 58].

The numerical treatment of evolution equations and related problems is challenging. For instance, the discretization of associated optimal control problems leads to large-scale optimization problems that are highly expensive to solve. This is especially true for nonlinear equations. Therefore, model order reduction-techniques (MOR) play an important role in this context and other many-query scenarios, i.e.

Fabian Hoppe

hoppe@ins.uni-bonn.de

Ira Neitzel

neitzel@ins.uni-bonn.de

1 Institut für Numerische Simulation, Rheinische Friedrich-Wilhelms-Universität Bonn,

Endenicher Allee 19b, 53115 Bonn, Germany 
in situations where the same equation has to be solved repeatedly for different right hand sides or parameters. The aim of MOR is to replace the high-dimensional original model by a suitable model with less degrees of freedom, the so-called reducedorder model. A prominent method of MOR for parabolic PDEs is the so-called Proper Orthogonal Decomposition (POD) method, [64]. This approach uses socalled snapshots of the dynamical system to construct a low-dimensional subspace of e.g. a high-dimensional finite-element space. More generally speaking, projection of a high-dimensional dynamical or parametric system onto smaller dimensional spaces leads to so-called reduced basis methods (RB), see e.g. [31]. These subspaces need to be in some sense capable of expressing the original trajectory of the system sufficiently well. The question of estimating the model order reduction error arises naturally and has been subject to intensive research. We refer e.g. to [52] and the references therein for RB-methods in PDE-constrained optimization in general, and to [53] or the survey [29] for POD in particular.

Since there is a huge amount of literature about POD/RB-MOR, not just for uncontrolled equations but even in the context of PDE-constrained optimization, we have to restrict ourselves to an incomplete literature overview. POD-error-estimates have been obtained in the a-priori regime for linear parabolic equations in [39], and for certain nonlinear equations e.g. in [12, 40, 59]. For recently obtained RB-a-posteriori error-estimates for quasilinear equations with monotone nonlinearity related to magneto(quasi)statics, both elliptic and parabolic, we refer to [33, 34]. For a general nonlinear parabolic PDE including a-posteriori error estimation on the time-discrete level we exemplarily cite [19], and refer to its introduction for an overview over further literature. Moreover, let us mention some results related to optimal control: A-posteriori POD-errors for linear-quadratic optimal control problems have been derived in [63]. The technique has been extended to nonlinear problems in [38] and problems with mixed control-state constraints in [28]. POD-a-posteriori error-estimates for an optimal control problem with semilinear state equation with monotone nonlinearity have been discussed in [53]. Appropriate coupling between numerical optimization and MOR is an active area of research, see e.g. [26]. For approaches based on a-posteriori error estimation and trust-region type-algorithms, respectively, we refer to [7, 57], and in particular [52, 53]. A different method, so-called optimality system POD, has been proposed in [41]. A related aspect, the interplay between POD and discretization, is under consideration in e.g. [21-23]. Balancing of discretization- and POD-errors for an optimal control problem is addressed in [27]. Finally, we exemplarily cite [2] and [37] for RB/POD-MOR applied to robust and multiobjective optimization, respectively.

For quasilinear parabolic PDEs or related optimal control problems, even the analysis of the full-order model is less complete. We mention [30, 50] for the analysis of the equation itself based on the functional analytic tool of nonautonomous maximal parabolic regularity [5], and refer to [9, 10] for first- and second-order optimality conditions of related optimal control problems. For additional state constraints we refer to [36]. A quasilinear version of the so-called thermistor problem has been addressed in [48, 49], and convergence of the SQP method applied to the model problem from [9] has been proven in [35]. For earlier literature on quasilinear parabolic optimal control problems, and optimal control of quasilinear elliptic 
PDEs with refer to the introductions of $[9,10]$. Finite element discretization errorestimates for the state equation from [10] are obtained in [11]. Having in mind a possible coupling of numerical optimization and MOR à la $[52,53]$, we start in the present paper with deriving a-posteriori reduced basis errors for the corresponding state equation. Herein, the presence of a non-monotone nonlinearity is the main difference to earlier publications concerned with RB-a-posteriori error-estimates for nonlinear PDEs [33, 34, 53], and also poses the main difficulty in our analysis. Moreover, compared to $[19,33,52]$ also time-discretization errors are included in our estimates which may prevent the choice of unnecessarily accurate reduced-models below the time-discretization-error in practice. Nevertheless, our reference solution is semi-discrete (in space), i.e. we fix a spatial discretization and do not address errors arising from this.

The paper is organized as follows: We start by introducing the model problem and the underlying assumptions in Sect. 2. Further, we provide a short overview over the results obtained on this equation so far, and introduce its semi-discretization (in space), and the reduced-order counterpart thereof. Moreover, we provide a sketchy outlook how the results subsequently obtained might be applied in the context of PDE-constrained optimization. In Sect. 3 we prove RB-a-posteriori error-estimates for a reduced-order trajectory with certain time regularity. Depending on how much regularity of the semi-discrete (in space) solution we are willing to exploit, we present two different approaches to obtain a-posteriori error-estimates. In case that hyperreduction of the nonlinearity is done by empirical interpolation (EIM) we provide estimates that also include the additional EIM-error in Sect. 4. Finally, we illustrate our results by numerical experiments in Sect. 5 .

Notation Throughout the paper we follow the conventions of [9] and use standard notation for (Bochner-)Lebesgue, (Bochner-)Sobolev-, and Hölder-spaces. By subscript $D$ we denote incorporation of certain homogeneous Dirichlet boundary conditions. If the underlying spatial domain becomes clear from the context we will omit it, i.e. we write $H_{D}^{1}$ instead of $H_{D}^{1}(\Omega)$ for instance.

\section{Model problem, assumptions, and RB-MOR}

In this section we first introduce the continuous model equation with non-monotone nonlinearity, and recall some regularity results under appropriate assumptions. Second, we state the semi-discrete (in space) version and its RB-reduction. Finally, we briefly explain how the results obtained in this paper might be applied to an optimal control problem associated to the model equation.

\subsection{Model problem, assumptions, and regularity results}

Let us start with defining the setting for the following quasilinear parabolic PDE: 


$$
\left.\begin{array}{rlrl}
\partial_{t} u+\mathscr{A}(u) u & =f & \text { on } I \times \Omega, \\
u(0) & =u_{0} & & \text { on } \Omega .
\end{array}\right\}
$$

The nonlinear operator $\mathscr{A}$ is defined by

$$
\langle\mathscr{A}(u) \varphi, \psi\rangle_{H_{D}^{-1}, H_{D}^{1}}:=\int_{\Omega} \xi(u) \mu \nabla \varphi \nabla \psi \mathrm{d} x \mathrm{~d} t, \quad \varphi, \psi \in L^{2}\left(I, H_{D}^{1}\right),
$$

whenever $u: I \times \Omega \rightarrow \mathbb{R}$ is measurable. Boundary conditions are incorporated in the right hand side $f$ and the respective function spaces. The precise assumptions required for obtaining a well-posed problem are stated below.

Before going into the detailed assumptions, we would like to comment briefly on the non-monotone structure of the nonlinearity in (Eq). The main difficulty as well as the main novelty of this paper arise from this fact. Recall that a nonlinear operator $\mathscr{N}: X \rightarrow X^{*}$ on a Banach space $X$ is called monotone if

$$
\langle\mathscr{N}(x)-\mathscr{N}(y), x-y\rangle_{X^{*}, X} \geq 0 \quad \forall x, y \in X,
$$

and strongly monotone if there exists a constant $c>0$ such that

$$
\langle\mathscr{M}(x)-\mathscr{N}(y), x-y\rangle_{X^{*}, X} \geq c\|x-y\|_{X}^{2} \quad \forall x, y \in X,
$$

cf. e.g. [56, 65] for this notion and its application in the theory of nonlinear PDEs. It has turned out that exploitation of strong monotonicity of the nonlinear terms is also an important step in the derivation of RB-a-posteriori error-estimates for semilinear parabolic [53], quasilinear elliptic [34], and quasilinear parabolic [33] PDEs, respectively. Note that the quasilinear nonlinearities in [33, 34] refer to problems from magneto(quasi)statics and depend on the gradient of the solution. The nonlinear operator $H_{D}^{1} \rightarrow H_{D}^{-1}$ under consideration in the present paper, however, is given by the map $u \mapsto \mathscr{A}(u) u$, and hence it depends on the solution $u$ and not on its gradient $\nabla u$. The counterexample [20, Example 8.18] shows that this essentially changes the structure of the nonlinearity: It cannot be expected to be monotone, and therefore it is essentially different to those considered in [33, 34, 53]. In fact, the main difficulty in the derivation of RB-a-posteriori error-estimates will be to find a workaround for the missing strong monotonicity of our nonlinearity.

For the rest of this paper, we rely on the following minimal assumptions:

\section{Assumption 2.1}

1. $\Omega \subset \mathbb{R}^{d}, d \in\{1,2,3\}$, is a bounded domain with boundary $\partial \Omega . \Gamma_{N} \subset \partial \Omega$ is relatively open and denotes the Neumann boundary part, whereas $\Gamma_{D}=\partial \Omega \backslash \Gamma_{N}$ is the part of the boundary equipped with homogeneous Dirichlet conditions. By subscript $D$ we denote that a space of functions on $\Omega$ incorporates such homogeneous Dirichlet boundary conditions on $\Gamma_{D}$. We assume that $\Omega \cup \Gamma_{N}$ is Gröger regular [25] such that all chart-maps in the definition of Gröger regularity can be chosen volume-preserving. For some $T>0$ we define the time interval $I=[0, T]$. 
2. The function $\xi: \mathbb{R} \rightarrow \mathbb{R}$ is differentiable with bounded derivative, and $\mu: \Omega \rightarrow \mathbb{R}^{d \times d}$ is measurable, uniformly bounded, and coercive in the following sense:

$$
0<\mu_{\bullet}:=\inf _{x \in \Omega} \inf _{z \in \mathbb{R}^{d} \backslash\{0\}} \frac{z^{T} \mu(x) z}{z^{T} z}, \quad \mu^{\bullet}:=\sup _{x \in \Omega} \sup _{1 \leq i, j \leq d}\left|\mu_{i, j}(x)\right|<\infty .
$$

We assume a coercivity condition $0<\xi_{\bullet} \leq \xi \leq \xi^{\bullet}$ for $\xi$ as well. With this we define as above

$$
\langle\mathscr{A}(u) \varphi, \psi\rangle_{L^{2}\left(I, W_{D}^{1,2}\right)}:=\int_{I} \int_{\Omega} \xi(u) \mu \nabla \varphi \nabla \psi \mathrm{d} x \mathrm{~d} t, \quad \varphi, \psi \in L^{2}\left(I, W_{D}^{1,2}\right),
$$

whenever $u$ is a measurable function on $\Omega$.

3. We assume that there is some $p \in(d, 4)$ such that

$$
-\nabla \cdot \mu \nabla+1: W_{D}^{1, p} \rightarrow W_{D}^{-1, p}
$$

is a topological isomorphism. For $s>2$ such that $\frac{1}{s}<\frac{1}{2}\left(1-\frac{d}{p}\right)$ we choose

$$
f \in L^{s}\left(I, W_{D}^{-1, p}\right), \quad u_{0} \in\left(W_{D}^{-1, p}, W_{D}^{1, p}\right)_{1 / s^{\prime}, s} .
$$

It has been shown in [50, Theorem 5.3] that under these assumptions (Eq) is wellposed: There exists a unique solution $u$ with regularity

$$
u \in W^{1, s}\left(I, W_{D}^{-1, p}\right) \cap L^{s}\left(I, W_{D}^{1, p}\right) .
$$

In fact, this is even true for $\xi$ being locally instead of globally Lipschitz-continuous. In the present paper we focus on a semi-discrete formulation of (Eq) and its solution. For globally Lipschitz-continuous $\xi$, existence and sufficient regularity of such a semi-discrete solution is automatically ensured due to space-discretization, even without Gröger regularity of the domain or the isomorphism property in Assumption 2.1.3, see Proposition 2.2 and the introduction of Sect. 3.2 for details. Therefore, we keep the summary of results concerning (Eq) on the continuous level rather short: We refer for instance to [9, 36, 50] for a discussion of Assumption 2.1 and only recall two regularity results from the literature that might be seen as motivation for exploiting the respective regularity of the semi-discrete in space solution lateron: Regularity in case of right hand sides in the slightly more regular Bessel potentialspaces $H^{-\zeta, p}$ and appropriate initial regularity has been addressed in [9, section 3.2]. In particular, $C^{\alpha}\left(I, W_{D}^{1, p}\right)$-regularity of the solution with some $\alpha>0$ and $p \in(d, 4)$ is obtained under fairly general regularity assumptions that admit certain constellations of non-smooth domains and coefficients, mixed boundary conditions, and distributional right-hand sides $f$. An equation similar to the one in the present paper has been considered in [10, Theorem 2.3] for $C^{1,1}$-smooth domains and coefficients, homogeneous Dirichlet boundary conditions, integrable right-hand sides, and a possibly unbounded nonlinearity, including a semilinear term. The authors obtain $W^{2}$ 
-regularity results that enable the derivation of finite element error-estimates [11]. Applying their setting to $(\mathrm{Eq})$ yields $C^{0, \alpha}\left(I, W^{1, \infty}\right)$-regularity of the solutions with some $\alpha>0$, cf. also [36, Corollary 5.4].

\subsection{Semi-discretization in space and RB-MOR}

We now introduce the semi-discrete (in space) counterpart of (Eq). Its solution will serve as the so-called truth-solution, i.e. the reference solution to which the a-posteriori error-estimates will refer to. In particular, this means that we do not address spatial discretization errors in this paper. Moreover, we also introduce the RBreduced counterpart of the semi-discrete (in space) equation.

Let $V_{h}$ be an $H_{D}^{1}$-conforming finite element space on $\Omega$ and $I_{h}: H_{D}^{1} \rightarrow V_{h}$ be an appropriate interpolation operator. We may introduce the semi-discrete (in space) counterpart of (Eq) as follows: Find $u_{h} \in W^{1,2}\left(I, V_{h}^{*}\right) \cap L^{2}\left(I, V_{h}\right)$ such that

$$
\left.\begin{array}{r}
\left\langle\partial_{t} u_{h}(t), \varphi_{h}\right\rangle_{H_{D}^{-1}, H_{D}^{1}}+\left\langle\mathscr{A}\left(u_{h}(t)\right) u_{h}(t), \varphi_{h}\right\rangle_{H_{D}^{-1}, H_{D}^{1}}=\left\langle f(t), \varphi_{h}\right\rangle_{H_{D}^{-1}, H_{D}^{1}} \\
\forall t \in I, \varphi_{h} \in V_{h}, \\
u_{h}(0)=I_{h} u_{0} .
\end{array}\right\}
$$

Due to finite-dimensionality of $V_{h},\left(\mathrm{Eq}_{h}\right)$ results in a system of ordinary differential equations (ODEs) for the coefficients of $u_{h}$ w.r.t. some basis of $V_{h}$. This allows to discuss existence of solutions to $\left(\mathrm{Eq}_{h}\right)$, even without using all parts of Assumption 2.1.

Proposition 2.2 Assume that $V_{h}$ is a subspace of $C(\bar{\Omega})$. For any right-hand side $f \in L^{2}\left(I, H_{D}^{-1}\right)$ and initial value $I_{h} u_{0} \in V_{h}$ there exists a unique solution $u_{h}: I \rightarrow V_{h}$ of $\left(\mathrm{Eq}_{h}\right)$ such that

$$
\left\|u_{h}\right\|_{L^{\infty}\left(I, L^{2}\right) \cap L^{2}\left(I, H_{D}^{1}\right)}+\left\|u_{h}\right\|_{W^{1,2}\left(I, V_{h}^{*}\right) \cap L^{2}\left(I, V_{h}\right)} \leq C\left(\|f\|_{L^{2}\left(I, H_{D}^{-1}\right)}+\left\|I_{h} u_{0}\right\|_{L^{2}}\right),
$$

with a constant $C>0$ independent of $V_{h}$, f and $I_{h} u_{0}$.

For the rest of the paper we will refer to $u_{h}$ as the semi-discrete (in space) solution to $(\mathrm{Eq})$, or, shorter, the truth-solution. By $u$ we will denote the continuous in space and time solution to (Eq), short: the true solution. To our best knowledge, a finite element error analysis for $u_{h}$ in the setting of Assumption 2.1 without additional suppositions has not been carried out in the existing literature. We refer to the references in [11] or to the introduction of [16] for an overview of results under additional assumptions. Let us only mention two results: A $L^{\infty}\left(I, L^{2}\right) \cap L^{2}\left(I, H^{1}\right)$-quasibestapproximation result has been derived in [17] with a technique slightly related to the one applied in Sect. 3.2. In [16] for instance pointwise error-estimates have been obtained.

Proof Let $\left(\varphi_{i}\right)_{i=1, \ldots, N_{h}}, N_{h}=\operatorname{dim} V_{h}$, be a basis for $V_{h}$. Writing $u_{h}(t)=\sum_{i=1}^{N_{h}} \mathbf{x}_{i}(t) \varphi_{i}$, the coefficient vector $\mathbf{x}(t) \in \mathbb{R}^{N_{h}}$ fulfills 


$$
\mathbf{M} \partial_{t} \mathbf{x}(t)+\mathbf{A}(\mathbf{x}(t)) \mathbf{x}(t)=\mathbf{f}(t), \quad \mathbf{x}(0)=\mathbf{x}_{0},
$$

with

$$
\begin{aligned}
\mathbf{M} & :=\left(\left\langle\varphi_{i}, \varphi_{j}\right\rangle_{L^{2}}\right)_{i, j}, \\
\mathbf{A}(\mathbf{z}) & :=\left(\int_{\Omega} \xi\left(\sum_{n=1}^{N_{h}} \mathbf{z}_{n} \varphi_{n}\right) \mu \nabla \varphi_{i} \nabla \varphi_{j} d x\right)_{i, j} \\
\mathbf{f}(t) & :=\left(\left\langle f(t), \varphi_{i}\right\rangle_{H_{D}^{-1}, H_{D}^{1}}\right)_{i}
\end{aligned}
$$

and $\mathbf{x}_{0}$ being the coefficient vector of $I_{h} u_{0}$. Using continuity of the basis functions, as well as global Lipschitz-continuity and boundedness of $\xi$, one easily verifies that (1) satisfies the assumptions of [61, Lemma 5.7]. Therefore, existence and uniqueness of a solution $\mathbf{x} \in W^{1,2}\left(I, \mathbb{R}^{N_{h}}\right)$ to (1) follows, which implies existence and uniqueness of a solution $u_{h} \in W^{1,2}\left(I, V_{h}\right)$ to $\left(\mathrm{Eq}_{h}\right)$. The estimate follows by standard techniques: Testing $\left(\mathrm{Eq}_{h}\right)$ with $\varphi_{h}=u_{h}$ and integrating by parts yields the first summand of the estimate. The second summand is obtained by testing with an arbitray $\varphi_{h} \in L^{2}\left(I, V_{h}\right)$.

Note that the embedding $V_{h} \hookrightarrow C(\bar{\Omega})$ is crucial in the previous argument: It ensures Lipschitz continuity of $\mathbf{A}(\cdot)$ and therefore existence of a solution to (1) via a generalized Picard-Lindelöf principle. If $V_{h}$ is a classical Lagrange finite element space on a polygonal (polyhedral) domain $\Omega$ equipped with a triangular (tetrahedral) mesh, this assumption is obviously fulfilled. However, we would like to point out that except for the assumptions from Proposition 2.2 we do not rely on further details of spatial discretization.

Performing expensive computations in the high-dimensional space $V_{h}$ can be avoided by application of the reduced basis approach: We replace the finite element space $V_{h}$ by a much smaller $n$-dimensional subspace $V_{h}^{n} \subset V_{h}$ that is related to the physical properties of the system and might be determined by the well-known POD approach [39, 64], for instance. Although our arguments do not rely on the particular choice of $V_{h}^{n}$ and therefore also cover general RB-methods, we clearly have in mind $V_{h}^{n}$ 's obtained by POD and also restrict our numerical experiments in Sect. 5 to this case. Having at hand a reduced ansatz-space $V_{h}^{n} \subset V_{h}$ and a suitable projection $P_{n}: V_{h} \rightarrow V_{h}^{n}$, usually the $L^{2}$ - or $H^{1}$-orthogonal projection, we introduce the reduced-order counterpart of $\left(\mathrm{Eq}_{h}\right)$ as follows: Find $u_{h}^{n} \in W^{1,2}\left(I,\left(V_{h}^{n}\right)^{*}\right) \cap L^{2}\left(I, V_{h}^{n}\right)$ such that

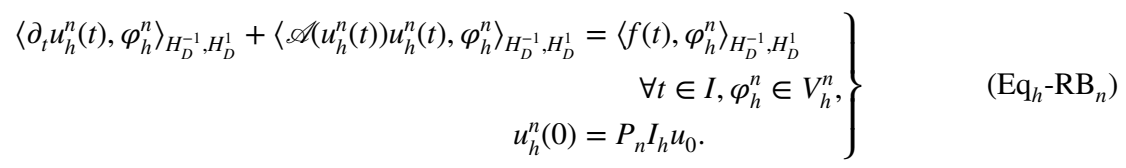

A reader familiar with ROM-techniques may already have noticed that the nonlinear term in (1) does not allow for efficient evaluation within the reduced-order model. 
We can overcome this issue by so-called hyperreduction techniques, e.g. the empirical interpolation method (EIM), which will be addressed in Sect. 4.

\subsection{Outlook towards optimal control}

In [9, Example 2.5] and [35] the following optimal control problem governed by (Eq) has been addressed:

$$
\left.\begin{array}{c}
\min _{u, q} J(u, q):=\frac{1}{2}\left\|u-u_{d}\right\|_{L^{2}(I \times \Omega)}^{2}+\frac{\gamma}{2}\|q\|_{L^{2}\left(I, \mathbb{R}^{k}\right)}^{2} \\
\text { s.t. } \quad q \in Q_{a d}:=\left\{q \in L^{2}\left(I, \mathbb{R}^{k}\right): q_{a} \leq q \leq q_{b} \text { a.e. on } I\right\}, \\
\text { and (Eq) with right-hand side } f=\sum_{i=1}^{k} q_{i} b_{i} .
\end{array}\right\}
$$

Hereby, $u_{d} \in L^{2}(I \times \Omega)$ denotes the desired state, $\gamma>0$ is a Tikhonov-parameter, $b_{i} \in H_{D}^{-\zeta, p} \subset H_{D}^{1}, i=1, \ldots, k$, are some fixed spatial control functions, and $q_{a}, q_{b} \in L^{\infty}\left(I, \mathbb{R}^{k}\right)$ define box-constraints for the control. The special structure of the right hand side $f$ is commonly referred to as purely time-dependent control in the literature [44]. Fixing a space-discretization for (Eq) as described earlier in this section results in a semi-discrete (in space) counterpart $\left(\mathrm{OCP}_{h}\right)$ of $(\mathrm{OCP})$, which we may consider again as reference object. Proposition 2.2 shows that this problem is well-defined.

At this point, let us make some short remarks about discretization of optimal control problems: Besides the discretization of the state variable and the state equation one also has to decide how to treat the control variable. There are several wellestablished concepts addressing the latter issue, e.g. variational discretization [14, 32], piecewise linear [54, 55], and piecewise constant [6] control discretization. For respective a-priori finite element discretization error-estimates we refer e.g. to [46, 47] for linear-quadratic parabolic optimal control, or to [51] for a semilinear parabolic state equation, also including discussion of the purely time-dependent control setting. Due to the purely time-dependent control structure of (OCP), the semi-discrete (in space) problem $\left(\mathrm{OCP}_{h}\right)$ may be regarded as variational discretization of (OCP) when only space is discretized. We mention e.g. [53] for the same semi-discrete (in space) approach to a-posteriori POD-errors for an optimal control problem governed by a semilinear parabolic PDE, or [15] for semi-discrete (in space) finite element error-estimates for optimal control of the instationary Navier-Stokes equation. There are different possibilities to choose an appropriate time-discretization. Our estimates in particular apply to CG1 time-discretization, but an extension to e.g. DG0 (implicit Euler) discretization can be easily stated, see Remark 4.4.

In numerical algorithms for the solution of $\left(\mathrm{OCP}_{h}\right)$, we may have to evaluate the semi-discrete reduced functional $j(q):=J\left(u_{h}(q), q\right)$ where $u_{h}(q)$ denotes the solution of $\left(\mathrm{Eq}_{h}\right)$ associated with several control functions $q$. Since repeated evaluation of $j_{h}$ is costly, RB-MOR can be applied to $\left(\mathrm{Eq}_{h}\right)$. Therefore, and due to additional time-discretization, we only have the possibility to compute an 
approximate solution $u_{h}^{n, m}=u_{h}^{n, m}(q)$ instead of $u_{h}(q)$. A short computation shows that the resulting error in the reduced functional can be estimated as follows:

$$
\left|J\left(u_{h}^{n, m}, q\right)-J\left(u_{h}, q\right)\right| \leq\left[\frac{1}{2}\left\|u_{h}^{n, m}-u_{h}\right\|_{L^{2}\left(I, L^{2}\right)}+\left\|u_{h}^{n, m}-u_{d}\right\|_{L^{2}\left(I, L^{2}\right)}\right]\left\|u_{h}^{n, m}-u_{h}\right\|_{L^{2}\left(I, L^{2}\right)} .
$$

Consequently, we immediately obtain an a-posteriori error for the reduced functional of $\left(\mathrm{OCP}_{h}\right)$ if we have an $L^{2}\left(I, L^{2}\right)$-estimate for solutions of $\left(\mathrm{Eq}_{h}\right)$ at hand. This may be regarded as motivation for the results in the present paper. Note that the above estimate for the functional error differs from the estimates obtained in [52, Theorems 4 and 9] because we do not utilize adjoint information. Deriving a-posteriori reduced modeling errors for the adjoint equation of $\left(\mathrm{OCP}_{h}\right)$, and consequently for the gradient of the reduced functional, is beyond the scope of the present paper. We refer to $[52,53]$ for such estimates in case of different model problems.

\section{A-posteriori RB-error-estimates}

In this section we state and prove our first main results: A-posteriori error-estimates for $\left(\mathrm{Eq}_{h}\right)$ including both reduced-order and time-discretization errors. For the reason of clarity we exclude hyperreduction for the nonlinearity at this point, and address this issue in the following section.

We roughly follow the ansatz of [53], where a semilinear equation with monotone nonlinearity has been discussed. To overcome the difficulties arising from the fact that our nonlinearity is not monotone we present two different approaches: The first approach, see Sect. 3.2, relies on exploiting $L^{\infty}\left(I, W^{1, \infty}\right)$-regularity of the truth-solution $u_{h}$ and allows to obtain explicit estimates of "classical" structure in terms of the error in the initial condition and the $V_{h}^{*}$-residual of the discrete solution under consideration. As a semi-discrete in space solution, $u_{h}$ obviously exhibits the required regularity for any fixed (spatial) discretization level. However, since the error-estimates will depend on the value of the $L^{\infty}\left(I, W^{1, \infty}\right)$-norm of $u_{h}$ it is desirable to have uniform bounds for this norm for all sufficiently fine spatial discretization levels. We believe that we can only expect such a uniform bound if the continuous in space and time solution of (Eq) exhibits $L^{\infty}\left(I, W^{1, \infty}\right)$-regularity, which is guaranteed in the setting of [10]. However, in the less regular setting of [9] we cannot expect such a result. Therefore, the second approach, see Sect. 3.3, is motivated by the intention to exploit less regularity of the truth-solution, more precisely: $L^{\infty}\left(I, W^{1, p}\right)$-regularity for some $p>d$. For continuous in space and time solutions of (Eq) this regularity is guaranteed in the setting of [9]. The price to pay for exploiting less regularity of $u_{h}$ is that we do not obtain an explicit formula for the error-estimate; instead the evaluation of the estimate requires the solution of an ODE. Moreover, for technical reasons we require additional assumptions on time regularity of the residual and the size of the initial error. We start by fixing the following notation and assumptions: 


\section{Assumption 3.1}

1. Assume that $V_{h} \subset H_{D}^{1} \cap C(\bar{\Omega}) \cap W^{1, \infty}(\Omega)$ is an $N_{h}$-dimensional conforming finite element space, and $V_{h}^{n}$ a $n$-dimensional subspace of $V_{h}$. By $u_{h} \in W^{1,2}\left(I, V_{h}^{*}\right) \cap L^{2}\left(I, V_{h}\right)$ we denote the truth-solution, i.e. the unique solution to $\left(\mathrm{Eq}_{h}\right)$.

2. Moreover, let $u_{h}^{n} \in W^{1,2}\left(I,\left(V_{h}^{n}\right)^{*}\right) \cap L^{2}\left(I, V_{h}^{n}\right)$ be arbitrary. By $e_{h}^{n}:=u_{h}^{n}-u_{h}$ we denote the error with respect to the truth-solution.

3. We denote the global Lipschitz-constant of $\xi$ by $\left|\xi^{\prime}\right|_{\infty}$.

We have in mind the following situation: $u_{h}^{n}$ is the solution of a time-discrete counterpart of $\left(\mathrm{Eq}_{h}-\mathrm{RB}_{n}\right)$, and we want to estimate how good $u_{h}^{n}$ approximates the truth-solution $u_{h}$. Note that in order to ensure that $u_{h}^{n}$ meets the regularity requirements of Assumption 3.1 we have to choose a time-discretization for $\left(\mathrm{Eq}_{h}-\mathrm{RB}_{n}\right)$ that results in sufficiently regular solutions, e.g. the Crank-Nicolson scheme in its CG1DG0 Petrov-Galerkin form. Time-discrete solutions of $\left(\mathrm{Eq}_{h}-\mathrm{RB}_{n}\right)$ obtained by Discontinuous Galerkin time-discretization, e.g. backward Euler, do not fulfill Assumption 3.1. Since discontinuous time-discretization might be of particular interest in the context of PDE-constrained optimization we outline an approach to overcome this restriction in Remark 4.4.

\subsection{Some preliminary calculations}

In this subsection we follow the residual-based ansatz of [53] as far as possible without modification, i.e. up to the point where strong monotonicity of the nonlinearity would be required. From that point on we develop two different approaches that will be discussed in the following subsections.

First, we introduce the residual of $u_{h}^{n}$ by

$$
r_{h}^{n}(t):=\partial_{t} u_{h}^{n}(t)+\mathscr{A}\left(u_{h}^{n}(t)\right) u_{h}^{n}(t)-f(t) \in\left(V_{h}^{n}\right)^{*} \hookrightarrow H_{D}^{-1}, \quad t \in I .
$$

To keep notation short we will omit the argument " $t$ " in the following. A short computation utilizing $\left(\mathrm{Eq}_{h}\right)$ shows that

$$
\left\langle r_{h}^{n}, \varphi_{h}\right\rangle_{H_{D}^{-1}, H_{D}^{1}}=\left\langle\partial_{t} e_{h}^{n}, \varphi_{h}\right\rangle_{H_{D}^{-1}, H_{D}^{1}}+\left\langle\mathscr{A}\left(u_{h}^{n}\right) u_{h}^{n}-\mathscr{A}\left(u_{h}\right) u_{h}, \varphi_{h}\right\rangle_{H_{D}^{-1}, H_{D}^{1}}
$$

holds for all $\varphi_{h} \in V_{h}$. We consider $V_{h}$ as a vector space canonically equipped with the $H_{D}^{1}$-norm. Therefore, its dual $V_{h}^{*}$ is canonically equipped with the following norm:

$$
\left\|\ell_{h}\right\|_{V_{h}^{*}}:=\sup _{0 \neq \psi_{h} \in V_{h}} \frac{\left\langle\ell_{h}, \psi_{h}\right\rangle_{H_{D}^{-1}, H_{D}^{1}}}{\left\|\psi_{h}\right\|_{H_{D}^{1}}}=\sup _{0 \neq \psi_{h} \in V_{h}} \frac{\ell_{h}\left(\psi_{h}\right)}{\left\|\psi_{h}\right\|_{H_{D}^{1}}} .
$$

Note that this norm is not equal to the $H_{D}^{-1}$-norm, because we only test with elements $\psi_{h}$ from $V_{h}$ in (4). For later use we state the following observation: 
Lemma 3.2 Let Assumption 3.1 hold. Then the function $I \rightarrow \mathbb{R}, t \mapsto\left\|r_{h}^{n}(t)\right\|_{V_{h}^{*}}^{2}$ is well-defined a.e. and $L^{2}$-integrable.

Proof This follows from the definition of $r_{h}^{n}$ and the regularity assumed for $u_{h}^{n}$.

Plugging in $\varphi_{h}=e_{h}^{n}(t)$ for every fixed $t$ in (3), and using the classical integration by parts formula from [56, Remark 7.5] we obtain

$$
\frac{d}{d t} \frac{1}{2}\left\|e_{h}^{n}\right\|_{L^{2}}^{2}+\left\langle\mathscr{A}\left(u_{h}^{n}\right) u_{h}^{n}-\mathscr{A}\left(u_{h}\right) u_{h}, e_{h}^{n}\right\rangle_{H_{D}^{-1}, H_{D}^{1}}=\left\langle r_{h}^{n}, e_{h}^{n}\right\rangle_{H_{D}^{-1}, H_{D}^{1}}
$$

Note that the second summand on the left-hand side of (5) causes problems in our case: If the nonlinearity $u \mapsto \mathscr{A}(u) u$ was strongly monotone, we could proceed as done in [53] for a semilinear term and estimate as follows:

$$
\left\langle\mathscr{A}\left(u_{h}^{n}\right) u_{h}^{n}-\mathscr{A}\left(u_{h}\right) u_{h}, e_{h}^{n}\right\rangle_{H_{D}^{-1}, H_{D}^{1}} \geq c\left|e_{h}^{n}\right|_{H_{D}^{1}}^{2} .
$$

However, as pointed out in Sect. 2.1 such an estimate cannot be expected to hold true. We cannot even bound the term under consideration from below by zero. Therefore, we have to proceed in a different way and split the problematic term into a coercive part and a remainder as follows:

$$
\begin{aligned}
\langle\mathscr{A} & \left.\left(u_{h}^{n}\right) u_{h}^{n}-\mathscr{A}\left(u_{h}\right) u_{h}, u_{h}^{n}-u_{h}\right\rangle_{H_{D}^{-1}, H_{D}^{1}} \\
& =\int_{\Omega}\left(\xi\left(u_{h}^{n}\right) \mu \nabla u_{h}^{n}-\xi\left(u_{h}\right) \mu \nabla u_{h}\right) \nabla\left(u_{h}^{n}-u_{h}\right) \mathrm{d} x \\
& =\int_{\Omega} \xi\left(u_{h}^{n}\right) \mu \nabla e_{h}^{n} \nabla e_{h}^{n} \mathrm{~d} x+\int_{\Omega}\left(\xi\left(u_{h}^{n}\right)-\xi\left(u_{h}\right)\right) \mu \nabla u_{h} \nabla e_{h}^{n} \mathrm{~d} x \\
\geq & \xi . \mu \cdot\left|e_{h}^{n}\right|_{H_{D}^{1}}^{2}+\int_{\Omega}\left(\xi\left(u_{h}^{n}\right)-\xi\left(u_{h}\right)\right) \mu \nabla u_{h} \nabla e_{h}^{n} \mathrm{~d} x .
\end{aligned}
$$

Plugging this into (5) yields

$$
\begin{aligned}
& \frac{d}{d t} \frac{1}{2}\left\|e_{h}^{n}\right\|_{L^{2}}^{2}+\xi_{\bullet} \mu_{\bullet}\left|e_{h}^{n}\right|_{H_{D}^{1}} \\
& \quad \leq\left\langle r_{h}^{n}, e_{h}^{n}\right\rangle_{H_{D}^{-1}, H_{D}^{1}}-\int_{\Omega}\left(\xi\left(u_{h}^{n}\right)-\xi\left(u_{h}\right)\right) \mu \nabla u_{h} \nabla e_{h}^{n} \mathrm{~d} x,
\end{aligned}
$$

i.e. except for the remainder term that we have shifted to the right-hand-side we have preserved a similar structure as in [53]. Formula (6) will serve as the common basis for our two different approaches in the following subsections. The main challenge in both cases is to estimate the second summand on the right-hand side in (6) in such a way that Gronwall's Lemma or a similar comparison principle can be applied to the resulting inequality. 


\subsection{A-posteriori estimates-Approach I}

The approach of this subsection is closer to [53] than the second one, and relies on $L^{\infty}\left(I, W^{1, \infty}\right)$-regularity of the truth-solution. An analogous regularity assumption on the true solution $u$ and a similar estimate of the nonlinear term as below have been used in [17] in the context of finite element errors.

Theorem 3.3 Let Assumptions 2.1 and 3.1 hold, and let $c_{\text {Lip }}>0$ be such that

$$
\left|u_{h}(t)\right|_{W^{1, \infty}} \leq c_{\text {Lip }} \quad \forall t \in I .
$$

Moreover, let $\varepsilon, \eta>0$ be chosen such that

$$
\eta+\varepsilon\left|\xi^{\prime}\right|_{\infty} \mu^{\cdot} c_{\mathrm{Lip}}=\xi_{.} \mu_{\bullet}
$$

and define $\beta:=2\left(\frac{1}{2 \varepsilon}\left|\xi^{\prime}\right|_{\infty} \mu^{\bullet} c_{\mathrm{Lip}}+\xi_{.} \mu_{\bullet}\right)$. Then, the following a-posteriori errorestimates for $u_{h}^{n}$ hold:

$$
\begin{aligned}
\left\|e_{h}^{n}(t)\right\|_{L^{2}}^{2} \leq e^{\beta t}\left\|u_{h}^{n}(0)-u_{h}(0)\right\|_{L^{2}}^{2}+\eta^{-1} \int_{0}^{t} e^{\beta(t-s)}\left\|r_{h}^{n}(s)\right\|_{V_{h}^{*}}^{2} \mathrm{~d} s \\
\left\|e_{h}^{n}\right\|_{L^{2}\left(I, L^{2}\right)}^{2} \leq \beta^{-1}\left(e^{\beta T}-1\right)\left\|u_{h}^{n}(0)-u_{h}(0)\right\|_{L^{2}}^{2} \\
+\eta^{-1} \beta^{-1} \int_{0}^{T}\left(e^{\beta(T-t)}-1\right)\left\|r_{h}^{n}(t)\right\|_{V_{h}^{*}}^{2} \mathrm{~d} t . \\
\left\|e_{h}^{n}\right\|_{L^{2}\left(I, H_{D}^{1}\right)}^{2} \\
\quad \xi_{\bullet}^{-1} \mu_{\bullet}^{-1} e^{\beta T}\left\|u_{h}^{n}(0)-u_{h}(0)\right\|_{L^{2}}^{2} \\
+\xi_{\bullet}^{-1} \mu_{\bullet}^{-1} \eta^{-1} \int_{0}^{T} e^{\beta(T-t)}\left\|r_{h}^{n}(t)\right\|_{V_{h}^{*}}^{2} \mathrm{~d} t .
\end{aligned}
$$

Proof We proceed with the argument from the previous subsection. Starting with the estimate (6) we bound the remaining term of the nonlinearity in the following way:

$$
\left|\int_{\Omega}\left(\xi\left(u_{h}^{n}\right)-\xi\left(u_{h}\right)\right) \mu \nabla u_{h} \nabla e_{h}^{n} \mathrm{~d} x\right| \leq\left|\xi^{\prime}\right|_{\infty} \mu^{\cdot} c_{\text {Lip }}\left\|e_{h}^{n}\right\|_{L^{2}}\left\|e_{h}^{n}\right\|_{H_{D}^{1}} .
$$

Using $W^{1, \infty}$-regularity for $u_{h}$ we can estimate one of the $e_{h}^{n}$-factors in the $L^{2}$-norm, which would not be possible assuming only $W^{1, p}$-regularity for $u_{h}$ with some finite $p$. With the help of Young's inequality we arrive at 


$$
\begin{aligned}
\frac{d}{d t} \frac{1}{2}\left\|e_{h}^{n}\right\|_{L^{2}}^{2}+\xi_{\bullet} \mu_{\bullet}\left\|e_{h}^{n}\right\|_{H_{D}^{1}}^{2} \leq & \xi_{\bullet} \mu_{\bullet}\left\|e_{h}^{n}\right\|_{L^{2}}^{2}+\left\langle r_{h}^{n}, e_{h}^{n}\right\rangle_{H_{D}^{-1}, H_{D}^{1}} \\
& +\left|\xi^{\prime}\right|_{\infty} \mu^{\cdot} c_{\text {Lip }}\left(\frac{1}{2 \varepsilon}\left\|e_{h}^{n}\right\|_{L^{2}}^{2}+\frac{\varepsilon}{2}\left\|e_{h}^{n}\right\|_{H_{D}^{1}}^{2}\right)
\end{aligned}
$$

with some $\varepsilon>0$. Another application of Young's inequality yields

$$
\begin{aligned}
\frac{d}{d t} \frac{1}{2}\left\|e_{h}^{n}\right\|_{L^{2}}^{2}+\xi_{\bullet} \mu_{\bullet}\left\|e_{h}^{n}\right\|_{H_{D}^{1}}^{2} \leq & \left(\frac{1}{2 \varepsilon}\left|\xi^{\prime}\right|_{\infty} \mu^{\cdot} c_{\text {Lip }}+\xi_{\bullet} \mu_{\bullet}\right)\left\|e_{h}^{n}\right\|_{L^{2}}^{2}+\frac{1}{2 \eta}\left\|r_{h}^{n}\right\|_{V_{h}^{*}}^{2} \\
& +\left(\frac{\eta}{2}+\frac{\varepsilon}{2}\left|\xi^{\prime}\right|_{\infty} \mu^{\cdot} c_{\text {Lip }}\right)\left\|e_{h}^{n}\right\|_{H_{D}^{1}}^{2}
\end{aligned}
$$

with $\eta>0$. Now, choose $\eta, \varepsilon$ as in the statement of the theorem and obtain

$$
\frac{d}{d t} \frac{1}{2}\left\|e_{h}^{n}\right\|_{L^{2}}^{2}+\frac{1}{2} \xi_{\bullet} \mu_{\bullet}\left\|e_{h}^{n}\right\|_{H_{D}^{1}}^{2} \leq \beta \cdot \frac{1}{2}\left\|e_{h}^{n}\right\|_{L^{2}}^{2}+\frac{1}{2 \eta}\left\|r_{h}^{n}\right\|_{V_{h}^{*}}^{2},
$$

where we will use from now on the abbreviation $\beta=2\left(\frac{1}{2 \varepsilon}\left|\xi^{\prime}\right|_{\infty} \mu^{\circ} c_{\text {Lip }}+\xi_{.} \mu_{\text {. }}\right)$ to enhance readability. With the help of Gronwall's Lemma [18, Corollary 2] we obtain an a-posteriori estimate for the $L^{\infty}\left(I, L^{2}\right)$-error from this:

$$
\left\|e_{h}^{n}(t)\right\|_{L^{2}}^{2} \leq\left\|P_{n} I_{h} u_{0}-I_{h} u_{0}\right\|_{L^{2}}^{2} e^{\beta t}+\eta^{-1} \int_{0}^{t}\left\|r_{h}^{n}(s)\right\|_{V_{h}^{e}}^{2} e^{\beta(t-s)} \mathrm{d} s .
$$

The second summand thereof is integrated using integration by parts, i.e.

$$
\int_{0}^{T} e^{\beta t}\left(\int_{0}^{t} e^{-\beta s}\left\|r_{h}^{n}(s)\right\|_{V_{h}^{*}}^{2} \mathrm{~d} s\right) \mathrm{d} t=\beta^{-1} \int_{0}^{T}\left(e^{\beta(T-t)}-1\right)\left\|r_{h}^{n}(t)\right\|_{V_{h}^{*}}^{2} \mathrm{~d} t,
$$

and together with the first summand we obtain the $L^{2}\left(I, L^{2}\right)$-estimate (8). As in [53] the $L^{2}\left(I, H^{1}\right)$-estimate (9) is obtained from (11) by integrating with respect to time over $I$ and using (8).

It is possible to exploit less regularity of $u_{h}$ as we will outline in the following. The price to pay is that the constants in the modified estimates cannot be computed explicitely, in general. In fact, if $d=2$ and $u_{h} \in L^{\infty}\left(I, W^{1,4}\right)$, estimate (10) can be replaced by

$$
\left|\int_{\Omega}\left(\xi\left(u_{h}^{n}\right)-\xi\left(u_{h}\right)\right) \mu \nabla u_{h} \nabla e_{h}^{n} \mathrm{~d} x\right| \leq\left|\xi^{\prime}\right|_{\infty} \mu^{\cdot}\left\|e_{h}^{n}\right\|_{L^{4}}\left|u_{h}\right|_{W^{1,4}}\left|e_{h}^{n}\right|_{H^{1}} .
$$

In order to apply Gronwall's Lemma as before, one has to observe 


$$
\begin{aligned}
\left\|e_{h}^{n}\right\|_{L^{4}}\left|u_{h}\right|_{W^{1,4}}\left|e_{h}^{n}\right|_{H^{1}} & \leq \frac{1}{2 \epsilon}\left\|e_{h}^{n}\right\|_{L^{4}}^{2}\left|u_{h}\right|_{W^{1,4}}^{2}+\frac{\epsilon}{2}\left|e_{h}^{n}\right|_{H^{1}}^{2} \\
& \leq \frac{C_{L G N, 2}^{2}}{2 \epsilon}\left|u_{h}\right|_{W^{1,4}}^{2}\left\|e_{h}^{n}\right\|_{L^{2}}\left|e_{h}^{n}\right|_{H^{1}}+\frac{\epsilon}{2}\left|e_{h}^{n}\right|_{H^{1}}^{2},
\end{aligned}
$$

where Young's inequality with parameter $\epsilon>0$ has been used in the first, and the 2D-Ladyzhenskaya-Gagliardo-Nirenberg interpolation inequality

$$
\|\varphi\|_{L^{4}} \leq C_{L G N, 2}\|\varphi\|_{L^{2}}^{\frac{1}{2}} \||\varphi|_{W^{1,4}}^{\frac{1}{2}}, \quad \varphi \in W^{1,4},
$$

in the second step. Finally, a second application of Young's inequality, again with parameter $\epsilon$, allows to obtain

$$
\left\|e_{h}^{n}\right\|_{L^{4}}\left|u_{h}\right|_{W^{1,4}}\left|e_{h}^{n}\right|_{H^{1}} \leq \frac{C_{L G N, 2}^{4}}{8 \epsilon^{3}}\left|u_{h}\right|_{W^{1,4}}^{4}\left\|e_{h}^{n}\right\|_{L^{2}}^{2}+\epsilon\left|e_{h}^{n}\right|_{H^{1}}^{2} .
$$

In dimension $d=3$ one has to apply

$$
\|\varphi\|_{L^{4}} \leq C_{L G N, 3}\|\varphi\|_{L^{2}}^{\frac{1}{4}}|\varphi|_{W^{1,4}}^{\frac{3}{4}}, \quad \varphi \in W^{1,4},
$$

and Young's inequality with exponents 4 and $\frac{4}{3}$ to arrive at

$$
\left\|e_{h}^{n}\right\|_{L^{4}}\left|u_{h}\right|_{W^{1,4}}\left|e_{h}^{n}\right|_{H^{1}} \leq \frac{C_{L G N, 3}^{8}}{64 \epsilon^{8}}\left|u_{h}\right|_{W^{1,4}}^{8}\left\|e_{h}^{n}\right\|_{L^{2}}^{2}+\left(\frac{\epsilon}{2}+\frac{3 \epsilon^{\frac{4}{3}}}{4}\right)\left|e_{h^{n}}^{n}\right|_{H^{1}}^{2} .
$$

In both cases one can proceed similarly as in the proof before in order to prove estimates for $e_{h}^{n}$ of structure analogous to those in Theorem 3.3. The main difference is that the constants $C_{L G N, 2}, C_{L G N, 3}>0$, whose exact values are unknown in general, enter the estimates. For upper bounds of these constants we refer e.g. to [1, Theorem 7.3] and the references therein.

\subsection{A-posteriori estimates-Approach II}

We derive a-posteriori error-estimates that rely on $L^{\infty}\left(I, W_{D}^{1, p}\right)$-regularity for the truth-solution $u_{h}$ with some $p>d$, only. For technical reasons we will have to impose an additional assumption on time-regularity of the residual and the size of the initial error. We start with the following auxiliary result:

Lemma 3.4 Let Assumptions 2.1 and 3.1 hold, and let $\varepsilon, \eta>0$ satisfy

$$
\xi_{.} \mu_{\bullet}=\eta+\varepsilon \cdot \mu^{\bullet}\left(2 \xi^{\bullet}\right)^{1-\frac{2}{q}}\left|\xi^{\prime}\right|_{\infty}^{\frac{2}{q}} c_{p} .
$$

The error-function $t \mapsto\left\|e_{h}^{n}(t)\right\|_{L^{2}}^{2}$ satisfies the differential inequality

$$
\varphi^{\prime}(t) \leq \alpha \varphi(t)+\beta \varphi(t)^{r}+\gamma(t), \quad t \in I,
$$


with the constants $\alpha=2 \xi_{.} \mu_{\bullet}, \quad \beta=\varepsilon^{-1} \mu^{\bullet}\left(2 \xi^{\bullet}\right)^{1-\frac{2}{q}}\left|\xi^{\prime}\right|_{\infty}^{\frac{2}{q}} c_{p}, \quad$ the function $\gamma=\gamma(t)=\eta^{-1}\left\|r_{h}^{n}(t)\right\|_{V_{h}^{*}}^{2}$, and the exponent $r=\frac{2}{q}=1-\frac{2}{p} \in(0,1)$.

As already for the discussion of the equation on the continuous level, cf. [9, 50], boundedness of $\xi$ is essential in our argument below.

Proof We pick up the argument from Sect. 3.1. We start by estimating the second summand on the right-hand side of (6) as follows:

$$
\left|\int_{\Omega}\left(\xi\left(u_{h}^{n}\right)-\xi\left(u_{h}\right)\right) \mu \nabla u_{h} \nabla e_{h}^{n} \mathrm{~d} x\right| \leq \mu^{\bullet}\left\|\xi\left(u_{h}^{n}\right)-\xi\left(u_{h}\right)\right\|_{L^{q}}\left|u_{h}\right|_{W^{1, p}}\left|e_{h}^{n}\right|_{H^{1}}
$$

with $p^{-1}+q^{-1}+\frac{1}{2}=1$, i.e. $q=\frac{2 p}{p-2}$. Note that we define the $W^{1, p}$-semi-norm as follows:

$$
|\varphi|_{W^{1, p}}^{p}:=\int_{\Omega}|\nabla \varphi|_{2}^{p} d x=\int_{\Omega}\left(\sum_{i=1}^{d}\left(\frac{\partial \varphi}{\partial x_{i}}\right)^{2}\right)^{p / 2} \mathrm{~d} x .
$$

Next, we apply the well-known Riesz-Thorin interpolation-inequality

$$
\|f\|_{L^{q}} \leq\|f\|_{L^{\infty}}^{1-\frac{2}{q}}\|f\|_{L^{2}}^{\frac{2}{q}}, \quad f \in L^{\infty}, q \in(2, \infty),
$$

to $f=\xi\left(u_{h}^{n}\right)-\xi\left(u_{h}\right)$, use $\|f\|_{L^{\infty}} \leq 2 \xi^{\bullet} \quad$ and the Lipschitz-estimate $\left\|\xi\left(u_{h}^{n}\right)-\xi\left(u_{h}\right)\right\|_{L^{2}} \leq\left|\xi^{\prime}\right|_{\infty}\left\|e_{h}^{n}\right\|_{L^{2}}$, and arrive at

$$
\left|\int_{\Omega}\left(\xi\left(u_{h}^{n}\right)-\xi\left(u_{h}\right)\right) \mu \nabla u_{h} \nabla e_{h}^{n} \mathrm{~d} x\right| \leq \mu^{\bullet}\left(2 \xi^{\bullet}\right)^{1-\frac{2}{q}}\left|\xi^{\prime}\right|_{\infty}^{\frac{2}{q}}\left|u_{h}\right|_{W^{1, p}}\left\|e_{h}^{n}\right\|_{L^{2}}^{\frac{2}{q}}\left|e_{h^{n}}^{n}\right|_{H^{1}}
$$

Note that this is the point where uniform boundedness of the nonlinearity $\xi$ enters. As before, we will estimate the product of the last two factors by Young's inequality and move the $H^{1}$-semi-norm term to the left-hand side of (6) in order to obtain an ODE for the $L^{2}$-error. This is why we are not able to convert the $\left\|e_{h}^{n}\right\|_{L^{2}}^{2 / q}$-term to an $\left\|e_{h}^{n}\right\|_{L^{2}}^{2}$-term by application of Young's inequality, because we need to generate an $\left|e_{h}^{n}\right|_{H^{1}}^{2}$ term from the second factor, such that this term can be canceled by the lefthand side of (6).

Plugging (13) into (6) and using Young's inequality twice we obtain: 


$$
\begin{aligned}
\frac{d}{d t} \frac{1}{2}\left\|e_{h}^{n}\right\|_{L^{2}}^{2}+\xi_{\bullet} \mu_{\bullet}\left\|e_{h}^{n}\right\|_{H_{D}^{1}} \leq & \frac{\eta}{2}\left\|e_{h}^{n}\right\|_{H_{D}^{1}}^{2}+\frac{1}{2 \eta}\left\|r_{h}^{n}\right\|_{V_{h}^{*}}^{2}+\xi_{\bullet} \mu_{\bullet}\left\|e_{h}^{n}\right\|_{L^{2}}^{2} \\
& +\mu^{\cdot}\left(2 \xi^{\bullet}\right)^{1-\frac{2}{q}}\left|\xi^{\prime}\right|_{\infty}^{\frac{2}{q}}\left|u_{h}\right|_{W^{1, p}} \\
& \cdot\left(\frac{\varepsilon}{2}\left\|e_{h}^{n}\right\|_{H^{1}}^{2}+\frac{1}{2 \varepsilon}\left\|e_{h}^{n}\right\|_{L^{2}}^{\frac{4}{q}}\right) .
\end{aligned}
$$

Here, $\varepsilon, \eta>0$ are the parameters appearing in Young's inequality. Choosing them as in the statement of the lemma yields

$$
\begin{aligned}
& \frac{d}{d t}\left\|e_{h}^{n}\right\|_{L^{2}}^{2}+\xi_{\bullet} \mu_{\bullet}\left\|e_{h}^{n}\right\|_{H^{1}}^{2} \\
& \quad \leq \eta^{-1}\left\|r_{h}^{n}\right\|_{V_{h}^{*}}^{2}+2 \xi_{\bullet} \mu_{\bullet}\left\|e_{h}^{n}\right\|_{L^{2}}^{2}+\varepsilon^{-1} \mu^{\cdot}\left(2 \xi^{\bullet}\right)^{1-\frac{2}{q}}\left|\xi^{\prime}\right|_{\infty}^{\frac{2}{q}} c_{p}\left\|e_{h}^{n}\right\|_{L^{2}}^{4 / q},
\end{aligned}
$$

from which the claim follows.

Let us briefly comment on the rather challenging structure of (12): First, note that $r \in(0,1)$, i.e. the right-hand side in (12) only depends Lipschitz-continuously on $\varphi(t)$, if $\varphi(t)$ stays uniformly away from zero, which can be ensured for $\varphi(0)>0$ only. Moreover, the Lipschitz-constant on sets bounded uniformly away from zero increases, if $r$ gets smaller. The latter, however, is the case if $p>d$ gets smaller, i.e. if we exploit less regularity of the truth-solution. In other words: The smaller the initial error, and the less regularity of the truth-solution we use, the more ill-posed (12) becomes.

Theorem 3.5 Let Assumptions 2.1 and 3.1 hold, and let $p>d$ and $c_{p}>0$ such that

$$
\left|u_{h}(t)\right|_{W^{1, p}} \leq c_{p} \quad \forall t \in I .
$$

Moreover, we assume that the initial error does not vanish, i.e. $\left\|e_{h}^{n}(0)\right\|_{L^{2}}>0$, and that $t \mapsto\left\|r_{h}^{n}(t)\right\|_{V_{h}^{*}}^{2}$ is piecewise continuous on I. Let $\varepsilon, \eta>0$ be chosen such that

$$
\xi_{.} \mu_{\bullet}=\eta+\varepsilon \cdot \mu^{\bullet}\left(2 \xi^{\bullet}\right)^{1-\frac{2}{q}}\left|\xi^{\prime}\right|_{\infty}^{\frac{2}{q}} c_{p}
$$

holds. Given the constants $\alpha=2 \xi_{.} \mu_{\bullet}, \beta=\varepsilon^{-1} \mu^{\bullet}\left(2 \xi^{\bullet}\right)^{1-\frac{2}{q}}\left|\xi^{\prime}\right|_{\infty}^{\frac{2}{q}} c_{p}$, and $r=1-\frac{2}{p}$, let $\varphi: I \rightarrow[0, \infty)$ be the solution to

$$
\begin{aligned}
\varphi^{\prime}(t) & =\alpha \varphi(t)+\beta \varphi(t)^{r}+\eta^{-1}\left\|r_{h}^{n}(t)\right\|_{V_{h}^{*}}^{2}, \quad t \in I, \\
\varphi(0) & =\left\|u_{h}^{n}(0)-u_{h}(0)\right\|_{L^{2}}^{2} .
\end{aligned}
$$

Then the following a-posteriori error-estimates hold true:

$$
\left\|e_{h}^{n}(t)\right\|_{L^{2}}^{2} \leq \varphi(t), \quad \forall t \in I, \quad\left\|e_{h}^{n}\right\|_{L^{2}\left(I, L^{2}\right)}^{2} \leq \int_{0}^{T} \varphi(s) \mathrm{d} s,
$$




$$
\begin{aligned}
\left\|e_{h}^{n}\right\|_{L^{2}\left(I, H_{D}^{1}\right)}^{2} \leq & \frac{1}{\xi_{.} \mu_{\bullet}}\left(\left\|u_{h}^{n}(0)-u_{h}(0)\right\|_{L^{2}}^{2}+\eta^{-1}\left\|r_{h}^{n}\right\|_{L^{2}\left(I, V_{h}^{*}\right)}^{2}\right. \\
& \left.\alpha \int_{0}^{T} \varphi(s) d s+\beta \int_{0}^{T} \varphi(s)^{2 / q} \mathrm{~d} s\right) .
\end{aligned}
$$

Proof In order to apply [18, Theorem 44] to Lemma 3.4 we have to verify that $f(t, z)=\alpha z+\beta z^{r}+\eta^{-1}\left\|r_{h}^{n}(t)\right\|_{V_{h}^{*}}^{2}$ satisfies the required assumption, i.e. that given $\varphi_{0}>0$ there is $\varepsilon>0$ such that the initial value problem

$$
\varphi^{\prime}(t)=f(t, \varphi(t)), \quad \varphi(0)=\varphi_{0}+\delta,
$$

has a solution on the time interval $I$ as long as $\delta \in[0, \varepsilon]$. Existence of a local solution on some time interval $\left[0, T_{\max }\right)$ with $T_{\max } \in(0, T]$ is clear due to Peano's existence-theorem, since $t \mapsto\left\|r_{h}^{n}(t)\right\|_{V_{h}^{*}}^{2}$ is piecewise continuous on $I$ and $u \mapsto \alpha u+\beta u^{r}$ even admits a continuous extension $\mathbb{R} \rightarrow \mathbb{R}, u \mapsto \alpha u+\beta \operatorname{sign}(u)|u|^{r}$. Further, due to $\alpha>0, \beta>0$, and $\left\|r_{h}^{n}(t)\right\|_{V_{h}^{*}}^{2} \geq 0$ it is clear that $\varphi$ is monotone increasing for $\varphi_{0}>0$. The theory of ODEs shows that either $T_{\max }=T$ or $T_{\max }<T$ and $\varphi(t) \rightarrow \infty$ as $t \rightarrow T_{\max }$. We argue by contradiction that $T_{\max }<T$ is impossible and therefore assume that $T_{\max }<T$. Then, because of $\varphi(t) \rightarrow \infty$ as $t \rightarrow T_{\max }$, there is $t_{0} \in(0, T)$ such that $\varphi(t)>1$ for $t \geq t_{0}$. For $t \geq t_{0}$ it holds due to $r \in(0,1)$ that

$$
\varphi^{\prime}(t)=\alpha \varphi(t)+\beta \varphi(t)^{r}+\eta^{-1}\left\|r_{h}^{n}(t)\right\|_{V_{h}^{*}}^{2} \leq(\alpha+\beta) \varphi(t)+\eta^{-1}\left\|r_{h}^{n}(t)\right\|_{V_{h}^{*}}^{2}
$$

By Gronwall's Lemma [18, Corollary 2] we conclude that $\varphi(t)$ stays bounded on $I$, which contradicts the assumption $T_{\max }<T$. Therefore, all solutions $\varphi$ have to exist on the whole time interval $I$. Thus, we have shown the estimate for the $L^{\infty}\left(I, L^{2}\right)$ error, from which we immediately obtain the $L^{2}\left(I, L^{2}\right)$-error by integration. Following again [53] we integrate (12) to obtain (16).

Note that the additional assumption on the residual is fulfilled, if e.g. $u_{h}^{n}$ is piecewise $C^{1}$ w.r.t. time on $I$. An explicit comparison principle for (12) as in [18, Corollary 2] would allow to obtain also explicit formulas in the estimates of Theorem 3.5. Unfortunately, we only found such results in the literature for the special cases $\gamma \equiv 0$ or $\alpha=0$ [18, Theorems 21 and 23], that are not of interest in the present context.

\section{A-posteriori RB- and EIM-error-estimates}

It is a well-known issue in RB-methods that the evaluation of nonlinear terms such as $\xi(u)$ requires access to the full number of degrees of freedom. Since the reasoning behind MOR is to avoid such computations within the full model, alternatives have to be found. In order to allow for an efficient offline-online splitting, the evaluation of nonlinearities in the reduced-order model for $(\mathrm{Eq})$ needs to be done by methods of hyperreduction, e.g. the Empirical Interpolation Method (EIM, [8]). In this 
section we describe a very basic version of the latter technique applied to our model problem, and show how the additional errors can be incorporated in the a-posteriori error-estimates of Theorems 3.3 and 3.5 using the same technique as in [33, 34].

\subsection{Empirical interpolation of $\mathscr{A}$}

First, we introduce EIM as far as required for our purpose and as concise as possible. For details see e.g. $[8,64]$. In order to present the main idea as clearly as possible, we stick to the continuous setting and omit space-discretization; the generalization to finite element spaces with a nodal basis is straightforward. Given so-called snapshots $y_{1}, \ldots, y_{N} \in C(\bar{\Omega})$, and a tolerance tol $_{\mathrm{EIM}}>0$, determine via a Greedy procedure some functions $\Xi_{1}, \ldots, \Xi_{m} \in C(\bar{\Omega})$, and interpolation points $x_{1}, \ldots, x_{m} \in \bar{\Omega}$ such that

$$
\xi\left(y_{\ell}\left(x_{j}\right)\right)=\sum_{k=1}^{m} c_{\ell, k} \Xi_{k}\left(x_{j}\right), \quad \ell=1, \ldots, N, \quad j=1, \ldots, m .
$$

implies $\left\|\xi\left(y_{\ell}\right)-\sum_{k=1}^{m} c_{\ell, k} \Xi_{k}\right\|_{L^{\infty}} \leq \operatorname{tol}_{\mathrm{EIM}}$. For some $w \in C(\bar{\Omega})$ we define the EIMapproximation of $\xi(w)$ as

$$
\xi_{m}^{\mathrm{EIM}}(w)=\sum_{k=1}^{m} c_{k} \Xi_{k}
$$

where $c \in \mathbb{R}^{m}$ solves the $m \times m$-system $\xi\left(w\left(x_{j}\right)\right)=\sum_{k=1}^{m} c_{k} \Xi_{k}\left(x_{j}\right), j=1, \ldots, m$. With this we may introduce a RB-EIM-reduced counterpart of (Eq) as

$$
\left.\begin{array}{r}
\left\langle\partial_{t} u_{h}^{n, m}(t), \varphi_{h}^{n}\right\rangle_{H_{D}^{-1}, H_{D}^{1}}+\left\langle\mathscr{A}_{m}^{\mathrm{EIM}}\left(u_{h}^{n, m}(t)\right) u_{h}^{n, m}(t), \varphi_{h}^{n}\right\rangle_{H_{D}^{-1}, H_{D}^{1}}=\left\langle f(t), \varphi_{h}^{n}\right\rangle_{H_{D}^{-1}, H_{D}^{1}} \\
\forall t \in I, \varphi_{h}^{n} \in V_{h}^{n}, \\
u_{h}^{n, m}(0)=P_{n} I_{h} u_{0},
\end{array}\right\} \quad\left(\mathrm{Eq}_{h}-\mathrm{RB}_{n}-\mathrm{EIM}_{m}\right)
$$

where $\mathscr{A}_{m}^{\text {EIM }}$ denotes the EIM-reduced version of the nonlinear differential operator defined by

$$
\left\langle\mathscr{A}_{m}^{\mathrm{EIM}}(u) \varphi, \psi\right\rangle_{H_{D}^{-1}, H_{D}^{1}}:=\int_{\Omega} \xi_{m}^{\mathrm{EIM}}(u) \mu \nabla u \nabla \varphi \mathrm{d} x, \quad \varphi, \psi \in H_{D}^{1} .
$$

Note that there is an efficient online evaluation of $\mathscr{A}_{m}^{\mathrm{EIM}}$, because the stiffness-matrices associated to the operators $-\nabla \cdot \Xi_{k} \mu \nabla$ can be precomputed in the offline-phase. Therefore, we only have to deal with $m$ and $n$ degrees of freedom, respectively, when dealing with $\mathscr{A}_{m}^{\mathrm{EIM}}$. In the following we will denote the EIM-error by

$$
\Delta_{m}^{\mathrm{EIM}}(u):=\left\|\xi(u)-\xi_{m}^{\mathrm{EIM}}(u)\right\|_{L^{\infty}} .
$$

Results addressing a-priori convergence of EIM can be found e.g. in [8, 24, 45]. For sophisticated algorithmic coupling of model order reduction and hyperreduction we 
refer to $[19,60]$ for instance. Other kinds of hyperreduction include e.g. discrete empirical interpolation (DEIM, [13]), or dynamic mode decomposition (DMD, [3]).

\subsection{A-posteriori RB-error-estimates including the EIM-error}

In this section we extend the results from Sect. 3 by incorporating also EIM-errors: It is clear that $\mathscr{A}\left(u_{h}^{n}\right) u_{h}^{n}$, and therefore $r_{h}^{n}$, cannot be computed efficiently during the online-phase due to the fact that the assembly of the stiffness-matrix for $\mathscr{A}\left(u_{h}^{n}\right)$ requires us to use the full number of degrees of freedom. Hence, the estimates of Theorems 3.3 and 3.5 cannot be evaluated efficiently in the online-phase. Consequently, evaluation of $r_{h}^{n}$ has to be avoided. Instead, given an arbitrary $u_{h}^{n, m}$ fulfilling Assumption 3.1, we introduce the EIM-reduced residual $r_{h}^{n, m}$ of $u_{h}^{n, m}$ as

$$
r_{h}^{n, m}(t):=\partial_{t} u_{h}^{n, m}(t)+\mathscr{A}_{m}^{\mathrm{EIM}}\left(u_{h}^{n, m}(t)\right) u_{h}^{n, m}(t)-f(t) \in V_{h}^{*} \hookrightarrow H_{D}^{-1}, \quad t \in I .
$$

It is obvious, that $r_{h}^{n, m}$ allows an efficient online evaluation. It remains to show how the error $u_{h}^{n, m}-u_{h}$ to the truth-solution can be estimated in terms of $r_{h}^{n, m}$ instead of $r_{h}^{n}$. Since all changes in the arguments already known from Sect. 3 are straightforward utilizing the estimates (18) and (19) below, we omit the details and only state the results. As before, we will we omit the argument " $t$ " in the following. A short computation as in Sect. 3.1 shows that the RB-EIM-error $e_{h}^{n, m}:=u_{h}^{n, m}-u_{h}$ fulfills

$$
\begin{aligned}
\left\langle r_{h}^{n, m}, \varphi_{h}\right\rangle_{H_{D}^{-1}, H_{D}^{1}}= & \left\langle\partial_{t} e_{h}^{n, m}, \varphi_{h}\right\rangle_{H_{D}^{-1}, H_{D}^{1}}+\left\langle\mathscr{A}\left(u_{h}^{n, m}\right) u_{h}^{n, m}-\mathscr{A}\left(u_{h}\right) u_{h}, \varphi_{h}\right\rangle_{H_{D}^{-1}, H_{D}^{1}} \\
& +\left\langle\mathscr{A}_{m}^{\mathrm{EIM}}\left(u_{h}^{n, m}\right) u_{h}^{n, m}-\mathscr{A}\left(u_{h}^{n, m}\right) u_{h}^{n, m}, \varphi_{h}\right\rangle_{H_{D}^{-1}, H_{D}^{1}} .
\end{aligned}
$$

As before it follows:

$$
\begin{aligned}
\frac{d}{d t} \frac{1}{2}\left\|e_{h}^{n, m}\right\|_{L^{2}}^{2}+\xi_{.} \mu_{.}\left|e_{h}^{n, m}\right|_{H^{1}}^{2} \leq & \left\langle r_{h}^{n, m}, e_{h}^{n, m}\right\rangle_{H_{D}^{-1}, H_{D}^{1}} \\
& -\int_{\Omega}\left(\xi\left(u_{h}^{n, m}\right)-\xi\left(u_{h}\right)\right) \mu \nabla u_{h} \nabla e_{h}^{n, m} \mathrm{~d} x \\
& -\int_{\Omega}\left(\xi^{\operatorname{EIM}}\left(u_{h}^{n, m}\right)-\xi\left(u_{h}^{n, m}\right)\right) \mu \nabla u_{h}^{n, m} \nabla e_{h}^{n, m} \mathrm{~d} x .
\end{aligned}
$$

The second summand on the right-hand side can be estimated as in Sects. 3.2 and 3.3. The third summand is estimated as follows:

$$
\begin{aligned}
\left|\int_{\Omega}\left(\xi^{\mathrm{EIM}}\left(u_{h}^{n, m}\right)-\xi\left(u_{h}^{n, m}\right)\right) \mu \nabla u_{h}^{n, m} \nabla e_{h}^{n, m} \mathrm{~d} x\right| & \leq \Delta_{m}^{\mathrm{EIM}}\left(u_{h}^{n, m}\right) \mu^{\bullet}\left|u_{h}^{n, m}\right|_{H^{1}}\left\|e_{h}^{n, m}\right\|_{H^{1}} \\
& \leq \frac{1}{2 \delta} \Delta_{m}^{\mathrm{EIM}}\left(u_{h}^{n, m}\right) \mu^{\bullet}\left|u_{h}^{n, m}\right|_{H^{1}}^{2}+\frac{1}{2} \delta \Delta_{m}^{\mathrm{EIM}}\left(u_{h}^{n, m}\right) \mu^{\bullet}\left\|e_{h}^{n, m}\right\|_{H^{1}}^{2},
\end{aligned}
$$

where $\delta>0$ is the parameter in Young's inequality. With this, we are ready to state the modified versions of the two main results from Sect. 3, beginning with the modified version of Theorem 3.3: 
Theorem 4.1 Let Assumptions 2.1 and 3.1 hold, and let $c_{\mathrm{Lip}}>0$ such that

$$
\left|u_{h}(t)\right|_{W^{1, \infty}} \leq c_{\text {Lip }} \quad \forall t \in I .
$$

Given $u_{h}^{n, m}$, choose $\varepsilon, \eta, \delta>0$ such that

$$
\eta+\varepsilon\left|\xi^{\prime}\right|_{\infty} \mu^{\bullet} c_{\text {Lip }}+\delta \Delta_{m}^{\mathrm{EIM}} \mu^{\bullet}=\xi_{.} \mu_{\bullet}
$$

is satisfied with the EIM-error $\Delta_{m}^{\mathrm{EIM}}:=\sup _{t \in I} \Delta_{m}^{\mathrm{EIM}}\left(u_{h}^{n, m}(t)\right)$. Moreover, we introduce the constant $\beta:=2\left(\frac{1}{2 \varepsilon}\left|\xi^{\prime}\right|_{\infty} \mu^{\bullet} c_{\mathrm{Lip}}+\xi_{\bullet} \mu_{\bullet}\right)$. Then the following a-posteriori error-estimates for $u_{h}^{n, m}$ hold true:

$$
\begin{aligned}
\left\|e_{h}^{n, m}(t)\right\|_{L^{2}}^{2} \leq & e^{\beta t}\left\|u_{h}^{n, m}(0)-u_{h}(0)\right\|_{L^{2}}^{2} \\
& +\int_{0}^{t} e^{\beta(t-s)}\left(\eta^{-1}\left\|r_{h}^{n, m}(s)\right\|_{V_{h}^{*}}^{2}+\delta^{-1} \Delta_{m}^{\mathrm{EIM}} \mu^{\bullet}\left|u_{h}^{n, m}(s)\right|_{H^{1}}^{2}\right) \mathrm{d} s, \\
\left\|e_{h}^{n, m}\right\|_{L^{2}\left(I, L^{2}\right)}^{2} \leq & \beta^{-1}\left(e^{\beta T}-1\right)\left\|u_{h}^{n, m}(0)-u_{h}(0)\right\|_{L^{2}}^{2} \\
& +\beta^{-1} \int_{0}^{T}\left(e^{\beta(T-t)}-1\right)\left(\eta^{-1}\left\|r_{h}^{n, m}(t)\right\|_{V_{h}^{*}}^{2}+\delta^{-1} \Delta_{m}^{\mathrm{EIM}} \mu^{\bullet}\left|u_{h}^{n, m}(t)\right|_{H^{1}}^{2}\right) \mathrm{d} t \\
\left\|\xi^{-1} \mu_{\bullet}^{-1} e^{\beta T}\right\| u_{h}^{n, m}(0)-u_{h}(0) \|_{L^{2}}^{2} & \\
& +\xi_{\bullet}^{-1} \mu_{\bullet}^{-1} \int_{0}^{T} e^{\beta(T-t)}\left(\eta^{-1}\left\|r_{h}^{n, m}(t)\right\|_{V_{h}^{*}}^{2}+\delta^{-1} \Delta_{m}^{\mathrm{EIM}} \mu^{\cdot}\left|u_{h}^{n, m}(t)\right|_{H^{1}}^{2}\right) \mathrm{d} t
\end{aligned}
$$

We also fix the following simplified estimates, that are less sharp but exhibit a favorable structure: They are weighted sums of the initial $L^{2}$-error, the $L^{2}-V_{h}^{*}$-norm of the residual, and the EIM-error. This allows to determine the optimal choice of the parameters $\varepsilon, \eta, \delta$ for these simpler estimates.

Corollary 4.2 Under the assumptions of the previous theorem it holds: 


$$
\begin{aligned}
\left\|e_{h}^{n, m}(t)\right\|_{L^{2}}^{2} \leq & e^{\beta t}\left\|u_{h}^{n, m}(0)-u_{h}(0)\right\|_{L^{2}}^{2}+e^{\beta t} \eta^{-1} \int_{0}^{t}\left\|r_{h}^{n, m}(s)\right\|_{V_{h}^{*}}^{2} \mathrm{~d} s \\
& +\delta^{-1} e^{\beta t} \Delta_{m}^{\mathrm{EIM}} \mu^{\cdot} \int_{0}^{t}\left|u_{h}^{n, m}(s)\right|_{H^{1}}^{2} \mathrm{~d} s, \\
\left\|e_{h}^{n, m}\right\|_{L^{2}\left(I, L^{2}\right)}^{2} \leq & \beta^{-1}\left(e^{\beta T}-1\right)\left\|u_{h}^{n, m}(0)-u_{h}(0)\right\|_{L^{2}}^{2} \\
& +\beta^{-1}\left(e^{\beta T}-1\right)\left(\eta^{-1}\left\|r_{h}^{n, m}\right\|_{L^{2}\left(I, V_{h}^{*}\right)}^{2}+\delta^{-1} \Delta_{m}^{\mathrm{EIM}} \mu^{\bullet}\left|u_{h}^{n, m}\right|_{L^{2}\left(I, H^{1}\right)}^{2}\right), \\
\left\|e_{h}^{n, m}\right\|_{L^{2}\left(I, H_{D}^{1}\right)}^{2} \leq & e^{\beta T} \xi_{\bullet}^{-1} \mu_{\bullet}^{-1}\left\|u_{h}^{n, m}(0)-u_{h}(0)\right\|_{L^{2}}^{2} \\
& +e^{\beta T} \xi_{\bullet}^{-1} \mu_{\bullet}^{-1}\left(\eta^{-1}\left\|r_{h}^{n, m}\right\|_{L^{2}\left(I, V_{h}^{*}\right)}^{2}+\delta^{-1} \Delta_{m}^{\mathrm{EIM}} \mu^{\bullet}\left|u_{h}^{n, m}\right|_{L^{2}\left(I, H^{1}\right)}^{2}\right) .
\end{aligned}
$$

The same technique as in Sect. 3.3 yields the following result:

Theorem 4.3 Let Assumptions 2.1 and 3.1 hold, and let $p>d$ and $c_{p}>0$ such that

$$
\left|u_{h}(t)\right|_{W^{1, p}} \leq c_{p} \quad \forall t \in I .
$$

Moreover, we assume that the initial error does not vanish, i.e. $\left\|e_{h}^{n, m}(0)\right\|_{L^{2}}>0$, and that $t \mapsto\left\|r_{h}^{n}(t)\right\|_{V_{h}^{*}}^{2}$ is piecewise continuous on I. Choose $\varepsilon, \eta, \delta>0$ such that

$$
\xi_{.} \mu_{\bullet}=\eta+\varepsilon \cdot \mu^{\bullet}\left(2 \xi^{*}\right)^{1-\frac{2}{q}}\left|\xi^{\prime}\right|_{\infty}^{\frac{2}{q}} c_{p}+\delta \Delta_{m}^{\mathrm{EIM}} \mu^{\cdot}
$$

is satisfied for the EIM-error $\Delta_{m}^{\mathrm{EIM}}=\sup _{t \in I} \Delta_{M}^{\mathrm{EIM}}\left(u_{h}^{n, m}(t)\right)$. Given the constants $\alpha=2 \xi_{\bullet} \mu_{\bullet}, \beta=\varepsilon^{-1} \mu^{\bullet}\left(2 \xi^{\bullet}\right)^{1-\frac{2}{q}}\left|\xi^{\prime}\right|_{\infty}^{\frac{2}{q}} c_{p}$, and $r=1-\frac{2}{p}$, let $\varphi: I \rightarrow[0, \infty)$ be the solution to

$$
\begin{aligned}
\varphi^{\prime}(t) & =\alpha \varphi(t)+\beta \varphi(t)^{r}+\eta^{-1}\left\|r_{h}^{n, m}(t)\right\|_{V_{h}^{*}}^{2}+\delta^{-1} \Delta_{m}^{\mathrm{EIM}} \mu^{\bullet}\left|u_{h}^{n, m}(t)\right|_{H^{1}}^{2}, \quad t \in I, \\
\varphi(0) & =\left\|e_{h}^{n, m}(0)\right\|_{L^{2}}^{2} .
\end{aligned}
$$

Then the following a-posteriori error-estimates hold true:

$$
\begin{aligned}
&\left\|e_{h}^{n, m}(t)\right\|_{L^{2}}^{2} \leq \varphi(t), \quad \forall t \in I, \quad\left\|e_{h}^{n, m}\right\|_{L^{2}\left(I, L^{2}\right)}^{2} \leq \int_{0}^{T} \varphi(s) \mathrm{d} s, \\
&\left\|e_{h}^{n, m}\right\|_{L^{2}\left(I, H_{D}^{1}\right)}^{2} \leq \frac{1}{\xi_{\bullet} \mu_{\bullet}}\left(\left\|u_{h}^{n, m}(0)-u_{h}(0)\right\|_{L^{2}}^{2}+\eta^{-1}\left\|r_{h}^{n}\right\|_{L^{2}\left(I, V_{h}^{*}\right)}^{2}\right. \\
&\left.+\delta^{-1} \Delta_{m}^{\mathrm{EIM}} \mu^{\cdot}\left|u_{h}^{n, m}\right|_{L^{2}\left(I, H^{1}\right)}^{2}+\alpha \int_{0}^{T} \varphi(s) \mathrm{d} s+\beta \int_{0}^{T} \varphi(s)^{2 / q} \mathrm{~d} s\right) .
\end{aligned}
$$


Note that the error-estimates in the results above hold true without additional assumptions on the size of residuals and EIM-errors. In practice, however, in order to obtain an accurate reduced-order model and corresponding small errorestimates, it will be necessary to construct RB- and EIM-bases in such a way that the size of residuals and EIM-errors is balanced appropriately. We do not address this issue here and refer for instance to $[19,60]$.

Moreover, let us point out that the EIM-error $\Delta_{m}^{\mathrm{EIM}}\left(u_{h}^{n, m}\right)$ at $u_{h}^{n, m}$ cannot be computed without referring to the full number of degrees of freedom; however, computation of $\left\|\xi\left(u_{h}^{n, m}\right)-\xi_{m}^{\text {EIM }}\left(u_{h}^{n, m}\right)\right\|_{L^{\infty}}$ in the full degrees of freedom is still much cheaper than computation of the respective full stiffness-matrices associated with the nonlinear elliptic operator that would be required for the computation of $r_{h}^{n}$. In contrast, note that the $H^{1}$-semi-norm of $u_{h}^{n, m}$ required in Theorems 4.1 and 4.3 admits efficient online evaluation, because it is induced by a bilinear form whose matrix w.r.t. the basis of $V_{h}^{n}$ can be precomputed and saved. Similarly, also the weight-matrices for the evaluation of the EIM-reduced residual can be precomputed and saved in the offline-phase.

To conclude this section, we shortly outline a possibility to relax Assumption 3.1 (2) in order to allow error estimation also for a discontinuous in time trajectory.

Remark 4.4 Let $u_{h}^{n, m}$ e.g. be given as

$$
u_{h}^{n, m}:=\mathbf{1}_{\{0\}} U_{h, 0}^{n, m}+\sum_{\ell=1}^{N_{t}} \mathbf{1}_{\left(t_{\ell-1}, t_{\ell}\right]} U_{h, \ell}^{n, m}, \quad U_{h, \ell}^{n, m} \in V_{h}^{n}, \quad \ell=0, \ldots, N_{t},
$$

for a partition $0=t_{0}<t_{1}<\cdots<t_{N_{t}-1}<t_{N_{t}}=T$. Such $u_{h}^{n, m}$ might be obtained by applying the backward Euler method in its DG0-formulation to $\left(\mathrm{Eq}_{h}-\mathrm{RB}_{n}-\mathrm{EIM}_{m}\right)$. Since our error-estimates do not apply directly to $u_{h}^{n, m}$ due to discontinuity w.r.t. time, we replace $u_{h}^{n, m}$ by its piecewise linear and continuous w.r.t time interpolation $\hat{u}_{h}^{n, m}$ w.r.t. the same partition defined by $\hat{u}_{h}^{n, m}\left(t_{\ell}\right):=u_{h}^{n, m}\left(t_{\ell}\right)=U_{h, \ell}^{n, m}$ for $\ell=0, \ldots, N_{t}$. Obviously, Theorems 4.1 and 4.3 apply to $\hat{u}_{h}^{n, m}$, and to obtain an estimate for the overall error we need to add the interpolation error $\hat{u}_{h}^{n, m}-u_{h}^{n, m}$. The latter can be computed explicitely:

$$
\begin{aligned}
\left\|u_{h}^{n, m}-\hat{u}_{h}^{n, m}\right\|_{L^{\infty}\left(I, L^{2}\right)}^{2} & \leq \max _{1 \leq \ell \leq N_{t}}\left\|U_{h, \ell}^{n, m}-U_{h, \ell-1}^{n, m}\right\|_{L^{2}}^{2}, \\
\left\|u_{h}^{n, m}-\hat{u}_{h}^{n, m}\right\|_{L^{2}\left(I, L^{2}\right)}^{2} & \leq \sum_{\ell=1}^{N_{t}} \frac{1}{3}\left(t_{\ell}-t_{\ell-1}\right)\left\|U_{h, \ell}^{n, m}-U_{h, \ell-1}^{n, m}\right\|_{L^{2}}^{2}, \\
\left\|u_{h}^{n, m}-\hat{u}_{h}^{n, m}\right\|_{L^{2}\left(I, H^{1}\right)}^{2} & \leq \sum_{\ell=1}^{N_{t}} \frac{1}{3}\left(t_{\ell}-t_{\ell-1}\right)\left\|U_{h, \ell}^{n, m}-U_{h, \ell-1}^{n, m}\right\|_{H^{1}}^{2} .
\end{aligned}
$$

The appearance of such jump-terms is what we may expect for an a-posteriori error for a discontinuous-in-time trajectory. Note that compared to classical a-posteriori error-estimates for discontinuous-in-time methods, see [42, 62] for instance, we do not assume that $u_{h}^{n, m}$ is the solution to a discrete-in-time analogue to $\left(\mathrm{Eq}_{h}-\mathrm{RB}_{n}\right)$. 


\section{Numerical illustration for POD-MOR}

In this final section of the paper we illustrate and compare the quality of our RBEIM-a-posteriori error-estimates numerically for three prototypical test problems. Although the results of this paper apply to general RB-methods, our particular focus is on POD-MOR. Therefore, we restrict ourselves to reduced ansatz-spaces $V_{h}^{n}$ spanned by a POD-basis of rank $n$ in our numerical tests.

\subsection{Test problems and technical details}

The two-dimensional domain $\Omega=[0,1]^{2}$ and the time interval $I=[0,1]$ are the same in all three test problems. We fix two discs $C_{1}=B_{\frac{1}{5}}\left(\frac{1}{4}, \frac{1}{4}\right)$ and $C_{2}=B_{\frac{1}{5}}\left(\frac{3}{4}, \frac{3}{4}\right)$, and the three boundary parts $\Gamma_{1}=\left\{x \in \partial \Omega: x_{2}=1\right\}$, $\Gamma_{2}=\left\{x \in \partial \Omega: x_{1}=0, x_{2}<\frac{1}{2}\right\}, \Gamma_{3}=\left\{x \in \partial \Omega: x_{1}=1, x_{2}<\frac{1}{2}\right\}$. The nonlinearity is given by

$$
\xi(u)=\frac{3}{4}+\frac{1}{2\left(1+e^{5 u}\right)} .
$$

We introduce the three test problems P1-P3 by equipping the equation

$$
\partial_{t} u-\nabla \cdot \xi(u) \nabla u=10 \sin (2 \pi t) \mathbf{1}_{C_{1}}-10 \cos (2 \pi t) \mathbf{1}_{C_{2}},
$$

with the following boundary and initial conditions, respectively:

(P1) Pure homogeneous Dirichlet boundary conditions and zero initial condition.

(P2) Pure homogeneous Neumann boundary conditions and zero initial condition.

(P3) Mixed boundary conditions: homogeneous Dirichlet boundary condition $u=0$ on $I \times \Gamma_{1}$, non-homogeneous Neumann conditions $\xi(u) \partial_{n} u=\sin (2 \pi t)$ on $I \times \Gamma_{2}$, and $\xi(u) \partial_{n} u=-\cos (2 \pi t)$ on $I \times \Gamma_{3}$, and natural boundary condition $\partial_{n} u=0$ on the remaining part of the boundary. The initial condition is $[u(0)]\left(x_{1}, x_{2}\right):=\frac{1}{10}\left(1-x_{1}\right)$.

Space- and time-discretization All computations are done utilizing FEniCS [4, 43] and piecewise linear finite elements on a mesh generated by mshr, the meshgeneration tool of EEniCS, with $N_{h}=5769$ degrees of freedom and maximum cell diameter $h_{\text {max }} \approx 2.1 \cdot 10^{-2}$. The POD-basis is generated with snapshots coming from an (implicit) Crank-Nicolson solution of the equation with $N_{t}=2500$ timesteps ("reference solution"). Hereby, the appearing nonlinear equations are solved by the built-in nonlinear solver of FEniCS. The same set of snapshots is also used to generate the EIM-approximation of the nonlinearity in a standard greedy procedure with $L^{\infty}$-tolerance $10^{-6}$ independent of the number of POD-basis functions, i.e. we do not balance accuracy of POD- and EIM-approximation. The POD-EIM-reduced equation is again solved utilizing the (implicit) Crank-Nicolson scheme with $N_{t}=2500$ timesteps, whereby the nonlinear algebraic equations appearing in every timestep are solved by a standard Newton-method that is initialized with a semi-implicit Euler step as first guess ("reduced solution"). Approximate true $L^{2}\left(I, L^{2}\right)-, L^{\infty}\left(I, L^{2}\right)$, and $L^{2}\left(I, H^{1}\right)$-errors are computed with respect to a further numerical solution that is 
computed on the same finite element mesh, but with a four times higher number of timesteps than for the snapshot generation ("truth-solution"). Finally, to ensure comparability between the different test problems and norms, all errors and estimates are relative errors, i.e. the absolute error or error-estimate is divided by the corresponding norm of the truth-solution.

Estimation of the required parameters Parameters like $\xi_{.}, \mu_{\bullet},\left|\xi^{\prime}\right|_{\infty}$ etc. are known from the problem data. The solution-dependent parameters are found as follows: The norms of $u_{h}$ are computed exactly based on the truth-solution in order to give the possibility to determine whether our estimates are sharp or not under the exact data. In real applications we would have to estimates those norms appropriately. The quality of the error-estimates -as absolute values- can heavily deteriorate in case of "safe" (i.e. large) estimates for the parameters. The same might happen in case of just inconvenient problem data due to the exponential terms in the estimates. However, we would like to point out that one might still hope in such a case that the relative behavior of the estimates, i.e. whether they decrease/increase by some factor, provides some information on the quality of the reduced model. Although we compute the EIM-error $\Delta_{m}^{\mathrm{EIM}}$ as defined in Sect. 4.1 by accessing the full number of degrees of freedom, we did not observe significant time consumption for this. We believe that this is due to the fact that evaluation of $\xi\left(u_{h}^{n, m}\right)$ in the full model is much cheaper than assembling the corresponding stiffness-matrices in the full model.

Choice of the exponent $p$ In order to obtain meaningful results we had to use relatively large values for $p$, e.g. $p=16$. Therefore, choosing $p$ according to the requirements of [9], i.e. only slightly larger than $d$ in general, seems to be difficult.

Estimates for Approach I (Theorem 4.1) For Approach I we determine the parameters $\varepsilon, \eta, \delta$ in such a way that the simpler estimates for the $L^{2}\left(I, H^{1}\right)$-error in Corollary 4.2 become optimal, and plug in the same parameters into the estimates from Theorem 4.1. Integrals with respect to time (residuals or weighted residuals in the formulas of Theorem 4.1) are evaluated using Gauss-quadrature of order 2 on every subinterval given by the timesteps.

Estimates for Approach II (Theorem 4.3) The following parameters turned out to be a good choice:

$$
\eta=\frac{1}{10}\left(1-\Delta_{m}^{\mathrm{EIM}}\right) \xi_{.} \mu_{\bullet}, \quad \varepsilon=\frac{9}{10} \frac{1-\Delta_{m}^{\mathrm{EIM}}}{\mu^{\bullet}\left(2 \xi^{\bullet}\right)^{1-\frac{2}{q}}\left|\xi^{\prime}\right|_{\infty}^{\frac{2}{q}} c_{p}}, \quad \delta=\frac{\xi_{\bullet} \mu_{\bullet}}{\mu^{\bullet}}
$$

Note that optimization of the parameters as in Approach I is not possible because we do not have an explicit formula at hand. The ODE for the evaluation of $\varphi$ is solved utilizing the backward difference formulae solver (BDF) within the solve ivp-routine from scipy. integrate, with relative tolerance $r$ tol $=10^{-6}$, and absolute tolerance atol $=10^{-3} \cdot\left\|u_{h}(0)-u_{h}^{n}(0)\right\|_{L^{2}}^{2}$. The maximal allowed step size is the same as the size of timesteps in the reduced model. We found that among other methods (Runge-Kutte with 2/3 and 4/5 stages, Radau) this choice delivered the best results. However, it is clear that the numerical approximation of $\varphi$ is challenging (in particular for small $p$ or small initial values), which might influence the reliability of the results. 
(a)

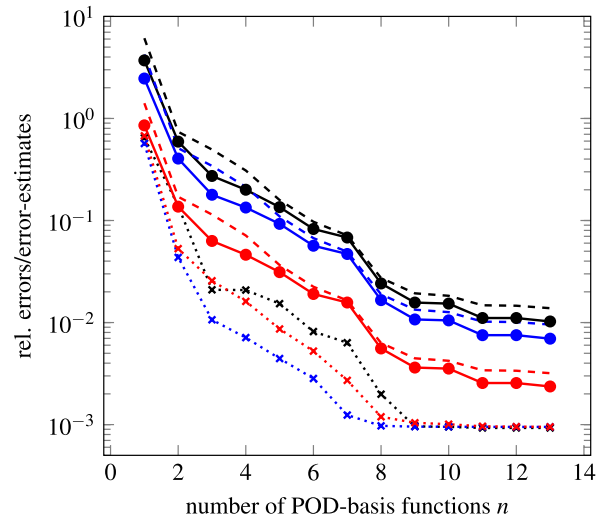

(b)

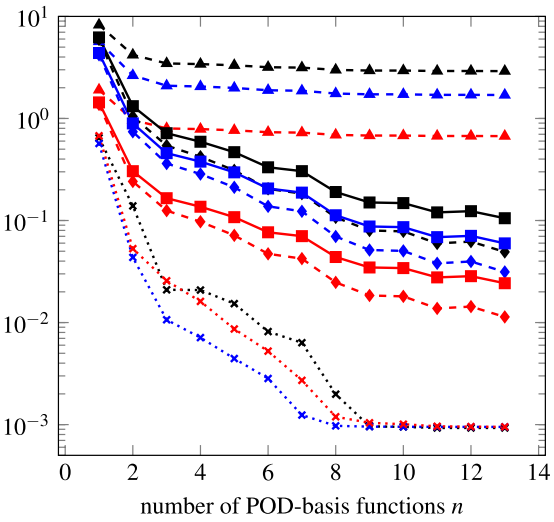

Fig. 1 Test problem P1 (homogeneous Dirichlet boundary conditions): a) Estimates from Approach I (•: Theorem 4.1 with optimized parameters, dashed lines: Corollary 4.2). b) Estimates from Approach II ( $(\mathbf{\Delta}$ : $p=6, \mathbf{m}: p=16, \boldsymbol{\gamma}, p=32) . L^{\infty}\left(I, L^{2}\right)-, L^{2}\left(I, L^{2}\right)$-, and $L^{2}\left(I, H^{1}\right)$-errors are displayed in black, blue, and red, respectively. Approximate true errors w.r.t. the truth-solution are included in dotted lines

(a)

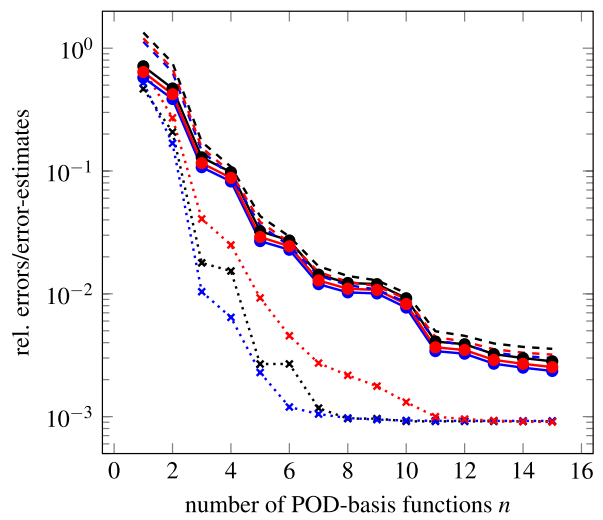

(b)

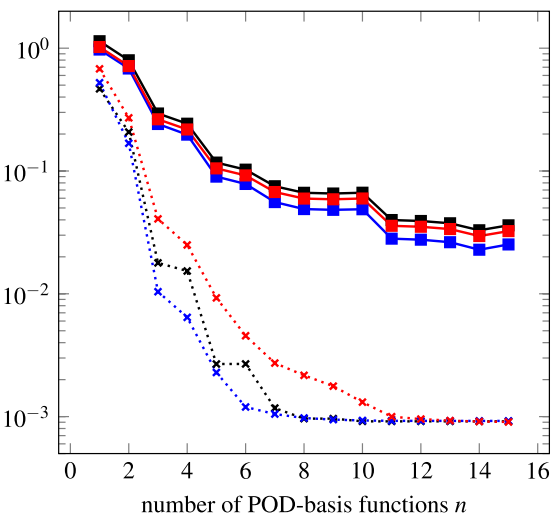

Fig. 2 Example P2 (homogeneous Neumann boundary conditions): a) Estimates from Approach I $(\bullet$ : Theorem 4.1 with optimized parameters, dashed lines: Corollary 4.2). b) Estimates from Approach II for $p=16$. $L^{\infty}\left(I, L^{2}\right)$-, $L^{2}\left(I, L^{2}\right)$-, and $L^{2}\left(I, H^{1}\right)$-errors are displayed in black, blue, and red, respectively. Approximate true errors w.r.t. the truth-solution are included in dotted lines

\subsection{Discussion of the results}

Figures 1, 2, 3 and 4 show the results of our experiments. It can be seen that Approach I yields better results the smoother the truth-solution is: Test problems P1 and P2 (homogeneous boundary conditions, Figs. 1 and 2) perform better than the problem with mixed boundary conditions (Test problem P3, Fig. 3). Moreover, we observe that the a-posteriori error-estimates of both approaches start stagnating 
(a)

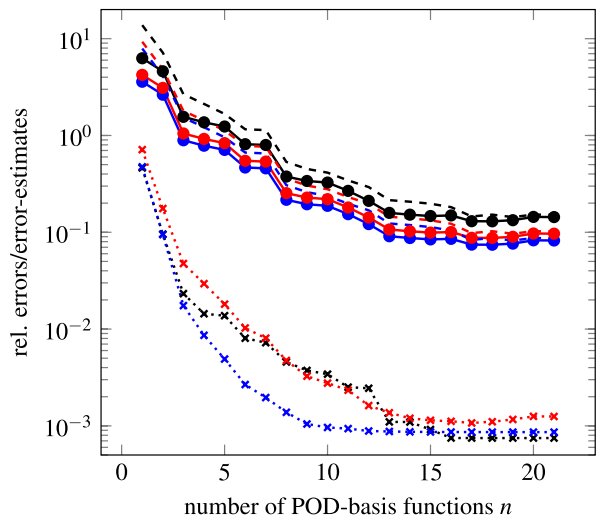

(b)

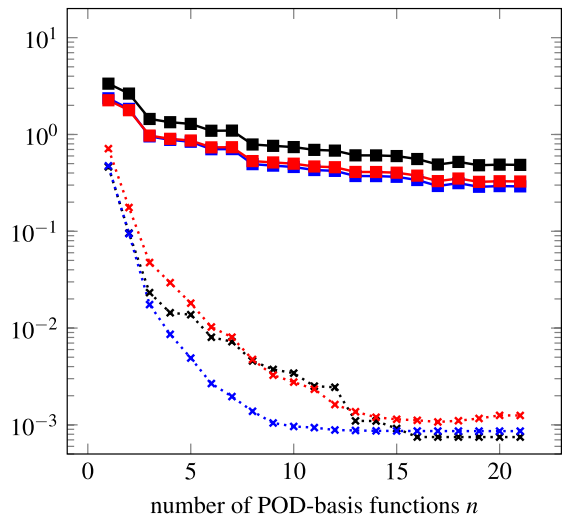

Fig. 3 Example P3 (mixed boundary conditions): a Estimates from Approach I (•: Theorem 4.1 with optimized parameters, dashed lines: Corollary 4.2). b Estimates from Approach II for $p=16 . L^{\infty}\left(I, L^{2}\right)$-, $L^{2}\left(I, L^{2}\right)$-, and $L^{2}\left(I, H^{1}\right)$-errors are displayed in black, blue, and red, respectively. Approximate true errors w.r.t. the truth-solution are included in dotted lines

Fig. 4 Error contributions in Example P3 (mixed boundary conditions): blue $\mathbf{m}$ : residual norms $\left\|r_{h}^{n}\right\|_{L^{2}\left(I, V_{h}^{*}\right)}$, black $\bullet$ : initial errors $\left\|e_{h}^{n}(0)\right\|_{L^{2}}$, red EIM-errors $\Delta^{\mathrm{EIM}}$ for $u_{h}^{n}$

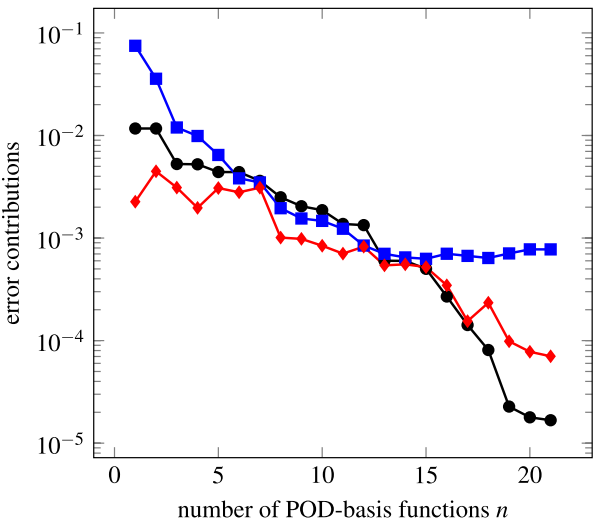

at about the same point at which also the true errors stagnate due to time-discretization. Indeed, in Fig. 4 it can be seen that this stagnation comes from stagnation of the residual norm at roughly the same magnitude as the size of time-steps. This indicates that from that point on the overall accuracy of the reduced-order model cannot be improved further by increasing the number of basis functions. See also e.g. [28] for balancing of POD-MOR- and time-discretization-errors for linear-quadratic parabolic optimal control problems.

How much Approach II depends on the choice of the exponent $p$ can be seen in Fig. 2b. The estimates stagnate very early for small $p$, i.e. Approach II unfortunately does not yield reasonable results in that case. For large $p$ the estimates seem to get closer to the values of Approach I. In this sense one might interpret Approach II as a modification of Approach I that trades strength of the required 
Table 1 Computing times for the setup of the EIM-reduction of the nonlinearity, the evaluation of the POD-EIM-reduced model, and the error-estimates, respectively

\begin{tabular}{llll}
\hline Computing times for & Example P1 & Example P2 & Example P3 \\
\hline number of EIM-basis functions & 28 & 36 & 49 \\
Setup EIM-reduced model & $50-57 \%$ & $70 \%$ & $99-157 \%$ \\
POD-EIM-reduced model & $1 \%(0.9 \%)$ & $1 \%(1.1 \%)$ & $1-4 \%(1.6 \%)$ \\
Approach I & $2-3 \%(3 \%)$ & $2-4 \%(4 \%)$ & $3-6 \%(5 \%)$ \\
Approach I optimized & $3-6 \%(6 \%)$ & $3-9 \%(8 \%)$ & $5-12 \%(10 \%)$ \\
Approach II & $3-15 \%(9-15 \%)$ & $4-22 \%(18 \%)$ & $6-15 \%(11 \%)$ \\
\hline
\end{tabular}

$100 \%$ correspond to the time that is required to compute the snapshots ("reference solution"). We show the range of times observed in the experiments from Figs. 1, 2 and 3, and in brackets we give the time observed for $n=13$ POD-basis functions

assumption (bigger $p$ means stronger assumption) against quality of results (smaller $p$ means less meaningful results and numerical instability).

For the computing times observed in our numerical experiments we refer to Table 1: The evaluation of the POD-EIM-reduced model is about 25- to 100-times faster than the evaluation of the full model. We believe that even higher speedups might be possible in case of finer finite element discretization. Compared to the computing time for the full model, evaluation of the a-posteriori error-estimates from Approach I is quite cheap: Evaluation of the POD-EIM-reduced model together with computation of an error-estimate still yields a speedup of factor at least 10. As expected, evaluation of the estimates from Approach II needs slightly more time.

Acknowledgements The authors thank the unknown referee for suggesting the alternative estimates in Sect. 3.2 based on the Ladyzhenskaya-Gagliardo-Nirenberg inequality.

Funding Open Access funding enabled and organized by Projekt DEAL. Funded by the Deutsche Forschungsgemeinschaft (DFG, German Research Foundation)—Projektnummer 211504053-SFB 1060.

Open Access This article is licensed under a Creative Commons Attribution 4.0 International License, which permits use, sharing, adaptation, distribution and reproduction in any medium or format, as long as you give appropriate credit to the original author(s) and the source, provide a link to the Creative Commons licence, and indicate if changes were made. The images or other third party material in this article are included in the article's Creative Commons licence, unless indicated otherwise in a credit line to the material. If material is not included in the article's Creative Commons licence and your intended use is not permitted by statutory regulation or exceeds the permitted use, you will need to obtain permission directly from the copyright holder. To view a copy of this licence, visit http://creativecommons.org/licen ses/by/4.0/.

\section{References}

1. Ahmad Ali, A., Deckelnick, K., Hinze, M.: Global minima for semilinear optimal control problems. Comput. Optim. Appl. 65(1), 261-288 (2016). https://doi.org/10.1007/s10589-016-9833-1 
2. Alla, A., Hinze, M., Kolvenbach, P., Lass, O., Ulbrich, S.: A certified model reduction approach for robust parameter optimization with PDE constraints. Adv. Comput. Math. 45(3), 1221-1250 (2019). https://doi.org/10.1007/s10444-018-9653-1

3. Alla, A., Kutz, J.N.: Nonlinear model order reduction via dynamic mode decomposition. SIAM J. Sci. Comput. 39(5), B778-B796 (2017). https://doi.org/10.1137/16M1059308

4. Alnæs, M.S., Blechta, J., Hake, J., Johansson, A., Kehlet, B., Logg, A., Richardson, C., Ring, J., Rognes, M.E., Wells, G.N.: The fenics project version 1.5. Arch. Num. Softw. 3(100) (2015). https:// doi.org/10.11588/ans.2015.100.20553

5. Amann, H.: Maximal regularity for nonautonomous evolution equations. Adv. Nonlinear Stud. 4(4), 417-430 (2004). https://doi.org/10.1515/ans-2004-0404

6. Arada, N., Casas, E., Tröltzsch, F.: Error estimates for the numerical approximation of a semilinear elliptic control problem. Comput. Optim. Appl. 23(2), 201-229 (2002). https://doi.org/10.1023/A: 1020576801966

7. Aria, E., Fahl, M., Sachs, E.: Trust-region proper orthogonal decomposition for flow control (2000)

8. Barrault, M., Maday, Y., Nguyen, N.C., Patera, A.T.: An 'empirical interpolation' method: application to efficient reduced-basis discretization of partial differential equations. C. R. Math. Acad. Sci. Paris 339(9), 667-672 (2004). https://doi.org/10.1016/j.crma.2004.08.006

9. Bonifacius, L., Neitzel, I.: Second order optimality conditions for optimal control of quasilinear parabolic equations. Math. Control Relat. Fields 8(1), 1-34 (2018). https://doi.org/10.3934/mcrf. 2018001

10. Casas, E., Chrysafinos, K.: Analysis and optimal control of some quasilinear parabolic equations. Math. Control Relat. Fields 8(3-4), 607-623 (2018). https://doi.org/10.3934/mcrf.2018025

11. Casas, E., Chrysafinos, K.: Numerical analysis of quasilinear parabolic equations under low regularity assumptions. Numer. Math. 143(4), 749-780 (2019). https://doi.org/10.1007/ s00211-019-01071-5

12. Chapelle, D., Gariah, A., Sainte-Marie, J.: Galerkin approximation with proper orthogonal decomposition: new error estimates and illustrative examples. ESAIM Math. Model. Numer. Anal. 46(4), 731-757 (2012). https://doi.org/10.1051/m2an/2011053

13. Chaturantabut, S., Sorensen, D.C.: Nonlinear model reduction via discrete empirical interpolation. SIAM J. Sci. Comput. 32(5), 2737-2764 (2010). https://doi.org/10.1137/090766498

14. von Daniels, N., Hinze, M., Vierling, M.: Crank-Nicolson time stepping and variational discretization of control-constrained parabolic optimal control problems. SIAM J. Control Optim. 53(3), 1182-1198 (2015). https://doi.org/10.1137/14099680X

15. Deckelnick, K., Hinze, M.: Semidiscretization and error estimates for distributed control of the instationary Navier-Stokes equations. Numer. Math. 97(2), 297-320 (2004). https://doi.org/10. 1007/s00211-003-0507-4

16. Dobrowolski, M.: $L^{\infty}$-convergence of linear finite element approximation to nonlinear parabolic problems. SIAM J. Numer. Anal. 17(5), 663-674 (1980). https://doi.org/10.1137/0717056

17. Douglas, J., Jr., Dupont, T.: Galerkin methods for parabolic equations. SIAM J. Numer. Anal. 7, 575-626 (1970). https://doi.org/10.1137/0707048

18. Dragomir, S.S.: Some Gronwall Type Inequalities and Applications. Nova Science Publishers Inc, Hauppauge, NY (2003)

19. Drohmann, M., Haasdonk, B., Ohlberger, M.: Reduced basis approximation for nonlinear parametrized evolution equations based on empirical operator interpolation. SIAM J. Sci. Comput. 34(2), A937-A969 (2012). https://doi.org/10.1137/10081157X

20. Francu, J.: Monotone operators. A survey directed to applications to differential equations. Apl. Mat. 35(4), 257-301 (1990)

21. Gräßle, C., Hinze, M.: POD reduced-order modeling for evolution equations utilizing arbitrary finite element discretizations. Adv. Comput. Math. 44(6), 1941-1978 (2018). https://doi.org/10. 1007/s10444-018-9620-x

22. Gräßle, C., Hinze, M., Lang, J., Ullmann, S.: POD model order reduction with space-adapted snapshots for incompressible flows. Adv. Comput. Math. 45(5-6), 2401-2428 (2019). https://doi. org/10.1007/s10444-019-09716-7

23. Gräßle, C., Hinze, M., Scharmacher, N.: POD for optimal control of the Cahn-Hilliard system using spatially adapted snapshots. In: Numerical Mathematics and Advanced ApplicationsENUMATH2017. Lecture Notes Computer Science and Engineering, vol. 126, pp. 703-711. Springer, Cham (2019). https://doi.org/10.1007/978-3-319-96415-7-65 
24. Grepl, M..A., Maday, Y., Nguyen, N..C., Patera, A..T.: Efficient reduced-basis treatment of nonaffine and nonlinear partial differential equations. M2AN Math. Model. Numer. Anal. 41(3), 575-605 (2007). https://doi.org/10.1051/m2an:2007031

25. Gröger, K.: A $W^{1, p}$-estimate for solutions to mixed boundary value problems for second order elliptic differential equations. Math. Ann. 283(4), 679-687 (1989). https://doi.org/10.1007/ BF01442860

26. Gräßle, C., Gubisch, M., Metzdorf, S., Rogg, S., Volkwein, S.: POD basis updates for nonlinear PDE control. Automatisierungstechnik 65(5), 298 - 307 (2017). https://doi.org/10.1515/ auto-2016-0100

27. Gubisch, M., Neitzel, I., Volkwein, S.: A-posteriori error estimation of discrete POD models for PDE-constrained optimal control. In: Model Reduction of Parametrized Systems. MS\&A. Model. Simul. Appl, vol. 17, pp. 213-234. Springer, Cham (2017)

28. Gubisch, M., Volkwein, S.: POD a-posteriori error analysis for optimal control problems with mixed control-state constraints. Comput. Optim. Appl. 58(3), 619-644 (2014). https://doi.org/10.1007/ s10589-014-9636-1

29. Gubisch, M., Volkwein, S.: Proper orthogonal decomposition for linear-quadratic optimal control. In: Model Reduction and Approximation. Computational Science and Engineering, vol. 15, pp. 3-63. SIAM, Philadelphia, PA (2017). https://doi.org/10.1137/1.9781611974829.ch1

30. Haller-Dintelmann, R., Rehberg, J.: Maximal parabolic regularity for divergence operators including mixed boundary conditions. J. Differ. Equ. 247(5), 1354-1396 (2009). https://doi.org/10.1016/j.jde. 2009.06.001

31. Hesthaven, J.S., Rozza, G., Stamm, B.: Certified reduced basis methods for parametrized partial differential equations. SpringerBriefs in Mathematics. Springer, Cham (BCAM Basque Center for Applied Mathematics, Bilbao) (2016). https://doi.org/10.1007/978-3-319-22470-1. BCAM SpringerBriefs

32. Hinze, M.: A variational discretization concept in control constrained optimization: the linear-quadratic case. Comput. Optim. Appl. 30(1), 45-61 (2005). https://doi.org/10.1007/s10589-005-4559-5

33. Hinze, M., Korolev, D.: A space-time certified reduced basis method for quasilinear parabolic partial differential equations. Adv. Comput. Math. 47(3), 36 (2021). https://doi.org/10.1007/ s10444-021-09860-z

34. Hinze, M., Korolev, D.: Reduced basis methods for quasilinear elliptic PDEs with applications to permanent magnet synchronous motors. Preprint. arXiv:2002.04288v1 (2020)

35. Hoppe, F., Neitzel, I.: Convergence of the SQP method for quasilinear parabolic optimal control problems. Optim. Eng. (2020). https://doi.org/10.1007/s11081-020-09547-2

36. Hoppe, F., Neitzel, I.: Optimal control of quasilinear parabolic PDEs with state constraints (2020) (Available as INS Preprint No. 2004)

37. Iapichino, L., Ulbrich, S., Volkwein, S.: Multiobjective PDE-constrained optimization using the reduced-basis method. Adv. Comput. Math. 43(5), 945-972 (2017). https://doi.org/10.1007/ s10444-016-9512-x

38. Kammann, E., Tröltzsch, F., Volkwein, S.: A posteriori error estimation for semilinear parabolic optimal control problems with application to model reduction by POD. ESAIM Math. Model. Numer. Anal. 47(2), 555-581 (2013). https://doi.org/10.1051/m2an/2012037

39. Kunisch, K., Volkwein, S.: Galerkin proper orthogonal decomposition methods for parabolic problems. Numer. Math. 90(1), 117-148 (2001). https://doi.org/10.1007/s002110100282

40. Kunisch, K., Volkwein, S.: Galerkin proper orthogonal decomposition methods for a general equation in fluid dynamics. SIAM J. Numer. Anal. 40(2), 492-515 (2002). https://doi.org/10.1137/S0036 142900382612

41. Kunisch, K., Volkwein, S.: Proper orthogonal decomposition for optimality systems. M2AN Math. Model. Numer. Anal. 42(1), 1-23 (2008). https://doi.org/10.1051/m2an:2007054

42. Liu, W., Ma, H., Tang, T., Yan, N.: A posteriori error estimates for discontinuous Galerkin timestepping method for optimal control problems governed by parabolic equations. SIAM J. Numer. Anal. 42(3), 1032-1061 (2004). https://doi.org/10.1137/S0036142902397090

43. Logg, A., Mardal, K.A., Wells, G.N., et al.: Automated Solution of Differential Equations by the Finite Element Method. Springer (2012). https://doi.org/10.1007/978-3-642-23099-8

44. de Los Reyes, J.C., Merino, P., Rehberg, J., Tröltzsch, F.: Optimality conditions for state-constrained PDE control problems with time-dependent controls. Control Cybernet. 37(1), 5-38 (2008)

45. Maday, Y., Mula, O., Turinici, G.: Convergence analysis of the generalized empirical interpolation method. SIAM J. Numer. Anal. 54(3), 1713-1731 (2016). https://doi.org/10.1137/140978843 
46. Meidner, D., Vexler, B.: A priori error estimates for space-time finite element discretization of parabolic optimal control problems. I. Problems without control constraints. SIAM J. Control Optim. 47(3), 1150-1177 (2008). https://doi.org/10.1137/070694016

47. Meidner, D., Vexler, B.: A priori error estimates for space-time finite element discretization of parabolic optimal control problems. II. Problems with control constraints. SIAM J. Control Optim. 47(3), 1301-1329 (2008). https://doi.org/10.1137/070694028

48. Meinlschmidt, H., Meyer, C., Rehberg, J.: Optimal control of the thermistor problem in three spatial dimensions, Part 1: existence of optimal solutions. SIAM J. Control Optim. 55(5), 2876-2904 (2017). https://doi.org/10.1137/16M1072644

49. Meinlschmidt, H., Meyer, C., Rehberg, J.: Optimal control of the thermistor problem in three spatial dimensions, Part 2: optimality conditions. SIAM J. Control Optim. 55(4), 2368-2392 (2017). https://doi.org/10.1137/16M1072656

50. Meinlschmidt, H., Rehberg, J.: Hölder-estimates for non-autonomous parabolic problems with rough data. Evol. Equ. Control Theory 5(1), 147-184 (2016). https://doi.org/10.3934/eect.2016.5. 147

51. Neitzel, I., Vexler, B.: A priori error estimates for space-time finite element discretization of semilinear parabolic optimal control problems. Numer. Math. 120(2), 345-386 (2012). https://doi.org/10. 1007/s00211-011-0409-9

52. Qian, E., Grepl, M., Veroy, K., Willcox, K.: A certified trust region reduced basis approach to PDEconstrained optimization. SIAM J. Sci. Comput. 39(5), S434-S460 (2017). https://doi.org/10.1137/ 16M1081981

53. Rogg, S., Trenz, S., Volkwein, S.: Trust-region POD using a-posteriori error estimation for semilinear parabolic optimal control problems, vol. 359. Technical Report (2017)

54. Rösch, A.: Error estimates for parabolic optimal control problems with control constraints. Z. Anal. Anwendungen 23(2), 353-376 (2004). https://doi.org/10.4171/ZAA/1203

55. Rösch, A.: Error estimates for linear-quadratic control problems with control constraints. Optim. Methods Softw. 21(1), 121-134 (2006). https://doi.org/10.1080/10556780500094945

56. Roubicek, T.: Nonlinear Partial Differential Equations with Applications, International Series of Numerical Mathematics, vol. 153. Birkhäuser Verlag, Basel (2005)

57. Schuh, M.: Adaptive Trust-Region POD Methods and Their Applications in Finance. Ph.D. thesis, University of Trier (2012)

58. Selberherr, S.: Analysis and Simulation of Semiconductor Devices. Springer-Verlag (1984)

59. Singler, J.R.: New POD error expressions, error bounds, and asymptotic results for reduced order models of parabolic PDEs. SIAM J. Numer. Anal. 52(2), 852-876 (2014). https://doi.org/10.1137/ 120886947

60. Smetana, K., Ohlberger, M.: Hierarchical model reduction of nonlinear partial differential equations based on the adaptive empirical projection method and reduced basis techniques. ESAIM Math. Model. Numer. Anal. 51(2), 641-677 (2017). https://doi.org/10.1051/m2an/2016031

61. Susu, L.M.: Analysis and Optimal Control of a Damage Model with Penalty. Ph.D. thesis, Fakultät für Mathematik der Technischen Universität Dortmund (2017)

62. Thomée, V.: Galerkin finite element methods for parabolic problems. Springer Series in Computational Mathematics, vol. 25, 2nd edn. Springer-Verlag, Berlin (2006)

63. Tröltzsch, F., Volkwein, S.: POD a-posteriori error estimates for linear-quadratic optimal control problems. Comput. Optim. Appl. 44(1), 83-115 (2009). https://doi.org/10.1007/s10589-008-9224-3

64. Volkwein, S.: Proper Orthogonal Decomposition: Theory and Reduced-Order Modelling. University of Konstanz, Lecture Notes (2012)

65. Zeidler, E.: Nonlinear functional analysis and its applications. II/B. Springer-Verlag, New York (1990). https://doi.org/10.1007/978-1-4612-0985-0 (Nonlinear monotone operators, Translated from the German by the author and Leo F. Boron)

Publisher's Note Springer Nature remains neutral with regard to jurisdictional claims in published maps and institutional affiliations. 